International Journal of Bifurcation and Chaos, Vol. 23, No. 3 (2013) 1350049 (18 pages)

(C) World Scientific Publishing Company

DOI: $10.1142 / \mathrm{S} 0218127413500491$

\title{
LOCAL BIFURCATION IN ONE-DIMENSIONAL NONAUTONOMOUS PERIODIC DIFFERENCE EQUATIONS
}

\author{
SABER ELAYDI \\ Department of Mathematics, Trinity University, \\ San Antonio, Texas, USA \\ selaydi@trinity.edu \\ RAFAEL LUÍS \\ Center for Mathematical Analysis, \\ Geometry and Dynamical System, Instituto Superior Técnico, \\ Technical University of Lisbon, Lisbon, Portugal \\ rafael.luis.madeira@gmail.com \\ HENRIQUE OLIVEIRA \\ Department of Mathematics, Instituto Superior Técnico, \\ Technical University of Lisbon, Lisbon, Portugal \\ holiv@math.ist.utl.pt
}

Received February 2, 2012; Revised December 2, 2012

\begin{abstract}
In this article we extend the theory of local bifurcation in one-dimensional autonomous maps to one-dimensional nonautonomous periodic maps. We give the necessary conditions for the main types of local bifurcation in one-dimensional periodic maps.
\end{abstract}

Keywords: Periodic maps; cycle; local bifurcation.

\section{Introduction}

The bifurcation theory for one-dimensional autonomous maps is well known and it may be found in many books on discrete dynamical systems (see, for instance [Cushing et al., 2003; Elaydi, 2008; Iooss, 1979; Robinson, 1999; Wiggins, 2003; Kuznetsov, 2004; Crawford, 1991; Hale \& Koçak, 1991]). In [Pötzsche, 2011b], the author stated that "it can be said that the bifurcation theory for autonomous discrete dynamical systems has reached a remarkable maturity w.r.t. analytical as well as numerical aspects and various effective computational tools are available". One of the most important software for numerical bifurcation analysis is the well-known MatCont [Dhooge et al., 2008].
One may speak about bifurcation when a small change in the parameter (the bifurcation parameter) of a system causes a sudden qualitative change in its behavior.

A saddle-node bifurcation occurs if, near the bifurcation point $\left(x^{*}, \mu^{*}\right)$, the map possesses a unique curve of fixed points in the $(\mu, x)$-plane which passes through the bifurcation point and lies on one side of the line $\mu=\mu^{*}$. A saddle-node bifurcation has the normal form $x_{n+1}=x_{n} \pm \mu \pm x_{n}^{2}$.

A transcritical bifurcation occurs if, near the bifurcation point $\left(x^{*}, \mu^{*}\right)$, the map possesses two curves of fixed points in the $(\mu, x)$-plane both of which pass through the bifurcation point and lie on both sides of the line $\mu=\mu^{*}$. An exchange 
of stability takes place at the bifurcation point. A transcritical bifurcation has the normal form $x_{n+1}=x_{n} \pm \mu x_{n} \pm x_{n}^{2}$.

A pitchfork bifurcation occurs if, near the bifurcation point $\left(x^{*}, \mu^{*}\right)$, the map possesses two curves of fixed points in the $(\mu, x)$-plane both of which pass through the bifurcation point and one of which lies on both sides of the line $\mu=\mu^{*}$. A pitchfork bifurcation has the normal form $x_{n+1}=x_{n} \pm \mu x_{n}-a x_{n}^{3}$, where for $a>0$ it is supercritical and for $a<0$ it is subcritical.

A period-doubling bifurcation occurs if, near the bifurcation point $\left(x^{*}, \mu^{*}\right)$, the map possesses a single curve of fixed points in the $(\mu, x)$ plane, while the second iterate $f^{2}$ undergoes a pitchfork bifurcation at the bifurcation point.

Notice that the normal form theory allows a classification of bifurcation scenarios by finding an algebraically most simple representation.

In the past few years many authors published relevant work on bifurcation theory for periodic nonautonomous discrete dynamical systems. Grinfeld et al. [1996] studied the bifurcation in twoperiodic logistic equation. AlSharawi and Angelos [2006] investigated the bifurcations of the periodically forced logistic map. D'Aniello and Oliveira [2009] studied the pitchfork bifurcation in twoperiodic discrete dynamical systems. Luís et al. [2010] studied the local bifurcation in two-periodic maps with Allee effect. Henson [1996] studied a local transcritical bifurcation where periodic solutions bifurcate from the trivial branch. In the latter work the period of the solution is the same as the period of the system. A related global version can be found in Cushing [1998]. Recently, Pötzsche [2012] investigated local and global bifurcations, as well as continuation properties for discrete-time periodic dynamical models in arbitrary (finite) dimension. Note that in this work the period of the solution is a multiple of the period of the system and Floquet theory yields the required linear theory.

Inspite of the growing literature on periodic difference equations, we are not able to find explicit results for one-dimensional nonautonomous periodic dynamical systems that cover the main types of bifurcations. This gap in the literature was also noticed by Pötzsche [2012].

It is the main objective of this paper to fill this gap in the current literature. Such study will have immediate applications to population models with periodically fluctuating habitats. We study the standard bifurcation for one-dimensional nonautonomous periodic difference equations, where the period of the solution is not necessarily a multiple of the period of the system. We will establish an extension of the theory of fixed points, that has been developed in the last four decades, from onedimensional autonomous discrete dynamical systems to one-dimensional nonautonomous periodic discrete dynamical systems, where the sequence of maps arises from a real one-parameter family of maps. Our techniques may be applied to $p$-periodic systems with only one dynamic variable, and one parameter for each individual map in the composition and $p$-parameters in the local bifurcations. The introduction of more parameters in each individual map and the very important case of global bifurcations are still works under progress.

It should be noted that the study of bifurcation in higher-dimensional systems will not be considered here. However, a remark is in order: In a forthcoming work, one of the authors of this paper, jointly with his collaborator (C. Pötzsche) studied a Neimark-Sacker bifurcation and center manifold reduction as well for periodic systems. Moreover, a continuation of the study done by Pötzsche [2012] considering any period of the solution will be taken into account.

In this paper we follow the techniques used for studying the bifurcation of autonomous difference equations (see for instance [Panfilov, 2005]). Hence, it is possible to apply the classical theory of bifurcations to the successive compositions of maps under consideration. First this vision would simplify a complex subject using the particular properties of each individual map in the iteration. Second, we study the periodic case, in a natural way, before the most general theory of bifurcation for nonperiodic discrete dynamical systems, where our techniques are not conceivable. The approach of studying only the compositions of maps gives rough results only for orbits of periods equal to the period of the system. Our approach, however, will study the invariance of the solutions of the bifurcation equations with any degree of degeneracy in terms of the cyclic permutations of iterations [Elaydi et al., 2011]. This can be done using a local topological conjugacy, which can be deduced from the local properties of each individual map in the iteration under some assumptions. These properties are almost impossible to treat using brute force 
methods on the composition of maps such as the use of the Fa-di-Bruno formula.

For nonautonomous bifurcations we refer the readers to the following studies: Rasmussen [2006] presents a survey in nonautonomous bifurcations in time-discrete equations. Hüls [2007] introduced a class of one-dimensional nonautonomous dynamical systems that allows an explicit study of its orbits as well as of the solutions of the associated variational equation. Furthermore, the solution operators also have explicit representations. In a special case, the model function can be transformed into the nonautonomous Beverton-Holt equation. Pötzsche [2010b] studied the local bifurcation properties for nonautonomous difference equations (and ordinary differential equations as well) using the fact that equilibria of periodic solutions typically do not exist and are replaced by bounded entire solutions as possible bifurcating objects. Here, exponential dichotomies in variation provide the necessary Fredholm theory in order to employ a LyapunovSchmidt reduction. Continuing this previous work, Pötzsche [2011a] provided local bifurcation properties using the fact that constant or periodic solutions are replaced by bounded entire solutions as bifurcating objects.

As a general source in nonautonomous dynamical systems, we refer the reader to two books in lecture notes [Rasmussen, 2007; Pötzsche, 2010a], the recent monograph [Kloedan \& Rasmussen, 2011b] and the survey paper [Kloeden et al., 2011a] with a focus on discrete dynamics.

Finally, we refer the readers to two papers in almost periodic difference equations. Hamaya [2004] studied bifurcation of almost periodic solutions of almost periodic difference equations using the Green's function for almost periodic operators and integral operators. Yet et al. [2011] studied nonautonomous saddle-node bifurcations in the quasiperiodically forced logistic map.

\section{Preliminaries}

A difference equation is called nonautonomous if it is governed by the rule

$$
x_{n+1}=f_{n}\left(x_{n}\right), \quad n \in \mathbb{Z}^{+},
$$

where $x \in X, X$ is a topological space. Here the orbit of a point $x_{0}$ is generated by the composition of the sequence of maps

$$
f_{0}, f_{1}, f_{2}, \ldots
$$

Explicitly,

$$
\begin{aligned}
x_{1} & =f_{0}\left(x_{0}\right), \\
x_{2} & =f_{1}\left(x_{1}\right)=f_{1} \circ f_{0}\left(x_{0}\right), \\
& \vdots \\
x_{n+1} & =f_{n} \circ f_{n-1} \circ \cdots \circ f_{1} \circ f_{0}\left(x_{0}\right),
\end{aligned}
$$

If the sequence of maps is periodic, i.e. $f_{n+p}=$ $f_{n}$, for all $n=0,1,2, \ldots$ and for some positive integer $p>1$, then we talk about nonautonomous periodic difference equations. Systems where the sequence of maps is periodic may model populations with fluctuation habitat, and they are commonly called periodically forced systems. Under this scenario, the dynamics is given by

$$
\begin{aligned}
x_{1} & =f_{0}\left(x_{0}\right), \\
x_{2} & =f_{1} \circ f_{0}\left(x_{0}\right), \\
& \vdots \\
x_{p} & =f_{p-1} \circ \cdots \circ f_{1} \circ f_{0}\left(x_{0}\right), \\
x_{p+1} & =f_{0} \circ f_{p-1} \circ \cdots \circ f_{1} \circ f_{0}\left(x_{0}\right), \\
x_{p+2} & =f_{1} \circ f_{0} \circ f_{p-1} \circ \cdots \circ f_{1} \circ f_{0}\left(x_{0}\right),
\end{aligned}
$$

Throughout this paper we work with nonautonomous periodic difference equations in which $p$ is the minimal period of Eq. (1) and $X=\mathbb{R}$.

Notice that the nonautonomous periodic difference equation given in (1) does not generate a discrete (semi)dynamical system [Elaydi \& Sacker, 2005a, 2005b] as it may not satisfy the (semi)group property. One of the most effective ways of converting the nonautonomous difference equation (1) into a discrete (semi)dynamical system is the construction of the associated skew-product system as described in a series of papers by Elaydi and Sacker [2005c, 2006]. It is noteworthy to mention that this idea was originally used to study nonautonomous differential equations by Sacker and Sell [1977].

Definition 2.1. An ordered set of points $C_{r}=\left\{\bar{x}_{0}\right.$, $\left.\bar{x}_{1}, \ldots, \bar{x}_{r-1}\right\}$ is an $r$-periodic cycle in $X$ if

$$
f_{(i+n r) \bmod p}\left(\bar{x}_{i}\right)=\bar{x}_{(i+1) \bmod r}, \quad n \in \mathbb{Z}^{+} .
$$

In particular,

$$
f_{i}\left(\bar{x}_{i}\right)=\bar{x}_{i+1}, \quad 0 \leq i \leq r-2,
$$


and

$$
f_{t}\left(\bar{x}_{t \bmod r}\right)=\bar{x}_{(t+1) \bmod r}, \quad r-1 \leq t \leq p-1 .
$$

It should be noted that the $r$-periodic cycle $C_{r}$ in $X$ generates an $s$-periodic cycle on the skewproduct $X \times Y\left(Y=\left\{f_{0}, f_{1}, \ldots, f_{p-1}\right\}\right)$ of the form

$$
\begin{aligned}
& \hat{C}_{s}=\left\{\left(\bar{x}_{0}, f_{0}\right),\left(\bar{x}_{1}, f_{1}\right), \ldots,\right. \\
&\left.\left(\bar{x}_{(s-1) \bmod r}, f_{(s-1) \bmod p}\right)\right\},
\end{aligned}
$$

where $s=l c m[r, p]$ is the least common multiple of $r$ and $p$.

To distinguish these two cycles, the $r$-periodic cycle $C_{r}$ on $X$ is called an $r$-geometric cycle (or simply $r$-periodic cycle when there is no confusion), and the $s$-periodic cycle $\hat{C}_{s}$ on $X \times Y$ is called an $s$-complete cycle. Notice that we may have $r<p$, $r=p$ or $r>p$.

The composition operator $\Phi$ is defined as follows

$$
\Phi_{n}^{i}=f_{n+i-1} \circ \cdots \circ f_{i+1} \circ f_{i} .
$$

When $i=0$ we write $\Phi_{n}^{0}$ as $\Phi_{n}$ and $\Phi_{0}=i d$.

As a consequence of the above remarks it follows that the $s$-complete cycle $\hat{C}_{s}$ is a fixed point of the composition operator $\Phi_{s}^{i}$. In other words, we have

$$
\Phi_{s}^{i}\left(\bar{x}_{i \bmod r}\right)=\bar{x}_{i \bmod r} .
$$

\section{Degeneracy}

In this section we study the genericity conditions of a generic discrete one-dimensional nonautonomous dynamical system that arises from a one-parameter family of maps.

Let

$$
\mathcal{F}=\left\{f_{0}, f_{1}, \ldots, f_{p-1}\right\}
$$

be a set of maps that arises from a one-parameter family of maps, i.e. each map is defined as

$$
f_{i}(x)=f\left(x, \mu_{i}\right),
$$

where $f_{i}: \mathbb{R} \rightarrow \mathbb{R}$. Moreover, we assume that the sequence of parameters is $p$-periodic, i.e. $\mu_{i}=$ $\mu_{i \bmod p}$. Under this assumption the one-dimensional difference equation (1) is $p$-periodic because

$$
f_{n}=f_{n+p} \quad \text { for all } n \in \mathbb{Z}^{+} .
$$

Moreover, the composition operator $\Phi_{s}^{i}$ is given by $\Phi_{s}^{i}: \mathbb{R} \times \mathbb{R}^{p} \rightarrow \mathbb{R}$, where

$$
\begin{aligned}
\Phi_{s}^{i}\left(\left(x, \mu_{i}, \mu_{i+1}, \ldots, \mu_{i+p-1}\right)\right) \\
=f_{s+i-1} \circ \cdots \circ f_{i+1} \circ f_{i}(x) \\
=f_{p+i-1} \circ \cdots \circ f_{i+1} \circ f_{i} \\
\quad \circ \cdots \circ f_{p+i-1} \circ \cdots \circ f_{i+1} \circ f_{i}(x)
\end{aligned}
$$

and $s$ is the least common multiple of $r$ and $p$.

We now recall the definition of genericity conditions. We will follow Kuznetsov's definition suggested in [Kuznetsov, 2004].

We say that a system satisfies a finite number of genericity conditions if

$$
N_{j}\left[\Phi_{s}^{i}\right] \neq 0, \quad j=1,2, \ldots, m,
$$

where each $N_{j}$ is some (algebraic) function of certain partial derivatives of $\Phi_{s}^{i}(x, \mu), x \in \mathbb{R}$ and $\mu=\left(\mu_{0}, \ldots, \mu_{p-1}\right) \in \mathbb{R}^{p}$, with respect to $x$ and $\mu$ evaluated at the fixed point $(x, \bar{\mu})=\left(\bar{x}_{i \bmod r}\right.$, $\left.\bar{\mu}_{i \bmod p}, \ldots, \bar{\mu}_{(p+i-1) \bmod p}\right)$ of the composition map $\Phi_{s}^{i}$. Otherwise, we say the map has a degeneracy condition.

There are two types of genericity conditions which are determined by a system of parameter values $\mu=\bar{\mu}$. The nondegeneracy conditions are the conditions that can be expressed in terms of partial derivatives of $\Phi_{s}^{i}(x, \bar{\mu})$ with respect to $x$ evaluated at $x=\bar{x}_{i \bmod r}$. All the other conditions, in which the partial derivatives of $\Phi_{s}^{i}(x, \mu)$ with respect to the parameters are involved, are called transversality conditions.

Before studying the bifurcation of periodic systems we present the following result which will be needed in the sequel.

Lemma 1. Consider the set of maps $\mathcal{F}=\left\{f_{0}\right.$, $\left.f_{1}, \ldots, f_{p-1}\right\}$ and let $C_{r}=\left\{\bar{x}_{0}, \bar{x}_{1}, \ldots, \bar{x}_{r-1}\right\}$ be an $r$-periodic cycle of the one-dimensional p-periodic difference equation (1). Then

$\frac{\partial \Phi_{s}}{\partial x}\left(\bar{x}_{0}\right)=\frac{\partial \Phi_{s}^{i}}{\partial x}\left(\bar{x}_{i \bmod r}\right), \quad$ for any $i=0,1,2, \ldots$, where $s$ is the least common multiple of $p$ and $r$.

Proof. If $i=0$ then there is nothing to prove. Using the chain rule it follows

$$
\begin{aligned}
\frac{\partial \Phi_{s}}{\partial x}\left(\bar{x}_{0}\right)= & \frac{\partial f_{s-1}}{\partial x}\left(\Phi_{s-1}\left(\bar{x}_{0}\right)\right) \times \frac{\partial f_{s-2}}{\partial x}\left(\Phi_{s-2}\left(\bar{x}_{0}\right)\right) \\
& \times \cdots \times \frac{\partial f_{0}}{\partial x}\left(\Phi_{0}\left(\bar{x}_{0}\right)\right)
\end{aligned}
$$




$$
\begin{aligned}
= & \frac{\partial f_{s-1}}{\partial x}\left(\bar{x}_{s-1 \bmod r}\right) \times \frac{\partial f_{s-2}}{\partial x}\left(\bar{x}_{s-2 \bmod r}\right) \\
& \times \cdots \times \frac{\partial f_{1}}{\partial x}\left(\bar{x}_{1 \bmod r}\right) \times \frac{\partial f_{0}}{\partial x}\left(\bar{x}_{0}\right) \\
= & \prod_{j=0}^{s-1} \frac{\partial f_{j}}{\partial x}\left(\bar{x}_{j \bmod r}\right) .
\end{aligned}
$$

(1) The first bifurcation condition holds

$$
\left.\frac{\partial \Phi_{s}}{\partial x}(x)\right|_{x=\bar{x}_{0}}=\prod_{j=0}^{s-1} \frac{d f_{j}}{d x}\left(\bar{x}_{j \bmod r}\right)=1 .
$$

(2) Higher degeneracy conditions hold for the composition operator $\Phi_{s}$

$$
\left.\frac{\partial^{n} \Phi_{s}}{\partial x^{n}}(x)\right|_{x=\bar{x}_{0}}=0, \quad n=2,3, \ldots
$$

Then the composition operator $\Phi_{s}^{i}$ satisfies

$$
\left.\frac{\partial^{n} \Phi_{s}^{i}}{\partial x^{n}}(x)\right|_{x=\bar{x}_{i \bmod r}}=0, \quad n=2,3, \ldots,
$$

for any $i=0,1,2, \ldots$.

We end this section presenting the following two results: the first is related to the transversality conditions and the second in higher degeneracy conditions on the parameters. The proofs will be omitted since it follows the guidelines in Lemma 1.

Lemma 3. Let $C_{r}=\left\{\bar{x}_{0}, \bar{x}_{1}, \ldots, \bar{x}_{r-1}\right\}$ be an $r$ periodic cycle of Eq. (1). Then for each $i=0$, $1,2, \ldots$ the following relations hold true

$$
\frac{\partial \Phi_{s}}{\partial x}\left(\bar{x}_{0}\right)=\prod_{j=2}^{s+1} \frac{\partial f_{j}}{\partial x}\left(\bar{x}_{j \bmod r}\right)=\frac{\partial \Phi_{s}^{2}}{\partial x}\left(\bar{x}_{2 \bmod r}\right) .
$$

Continuing this process, for any $i=0,1,2, \ldots$ one has

$$
\frac{\partial \Phi_{s}}{\partial x}\left(\bar{x}_{0}\right)=\frac{\partial \Phi_{s}^{1}}{\partial x}\left(\bar{x}_{1 \bmod r}\right)=\cdots=\frac{\partial \Phi_{s}^{i}}{\partial x}\left(\bar{x}_{i \bmod r}\right) .
$$

Hence, by the above observation, for the nondegeneracy conditions, it is enough to work with the derivative $\frac{\partial \Phi_{s}}{\partial x}\left(\bar{x}_{0}\right)$.

Recall that we have three possible situations: $r<p$, or $r=p$ or $r>p$. In any case, $\bar{x}_{i \bmod r}$ is a fixed point of the composition operator $\Phi_{s}^{i}$, where $s$ is the least common multiple of $r$ and $p$.

We now present a result in higher degeneracy conditions. The proof is analogous as above and will be omitted.

Lemma 2. Consider the set of maps $\mathcal{F}=\left\{f_{0}\right.$, $\left.f_{1}, \ldots, f_{p-1}\right\}$ and let $C_{r}=\left\{\bar{x}_{0}, \bar{x}_{1}, \ldots, \bar{x}_{r-1}\right\}$ be an $r$-periodic cycle of the one-dimensional p-periodic difference equation (1). Assume that the maps have a sufficient number of derivatives satisfying:

$$
\left.\frac{\partial \Phi_{s}}{\partial \mu_{j}}(x)\right|_{x=\bar{x}_{0}}=\left.\frac{\partial \Phi_{s}^{i}}{\partial \mu_{j}}(x)\right|_{x=\bar{x}_{i \bmod r}},
$$

for any $j \in\{0,1, \ldots, p-1\}$.

Lemma 4. Let $C_{r}=\left\{\bar{x}_{0}, \bar{x}_{1}, \ldots, \bar{x}_{r-1}\right\}$ be an $r$ periodic cycle of Eq. (1) and assume that the sequence of maps $f_{i}$ in $\mathcal{F}$ has a sufficient number of derivatives satisfying the conditions:

$$
\left.\frac{\partial \Phi_{s}}{\partial x}(x)\right|_{x=\bar{x}_{0}}=\prod_{j=0}^{s-1} \frac{d f_{j}}{d x}\left(\bar{x}_{j \bmod r}\right)=1 .
$$

(2) For each $j \in\{0,1, \ldots, p-1\}$

$$
\left.\frac{\partial^{n} \Phi_{s}}{\partial \mu_{j}^{n}}(x)\right|_{x=\bar{x}_{0}}=0, \quad n=2,3, \ldots
$$

Then, for each $j \in\{0,1, \ldots, p-1\}$ we have

$$
\left.\frac{\partial^{n} \Phi_{s}^{i}}{\partial \mu_{j}^{n}}(x)\right|_{x=\bar{x}_{i \bmod r}}=0, \quad n=2,3, \ldots
$$

for any $i=0,1,2, \ldots$. 


\section{Saddle-Node Bifurcation}

Since $\bar{x}_{0}$ is a fixed point of $\Phi_{s}\left(x, \mu_{0}, \mu_{1}, \ldots\right.$, $\left.\mu_{p-1}\right)$ we can work with the autonomous difference equation

$$
y_{n+1}=\Phi_{s}\left(y_{n}, \mu_{0}, \mu_{1}, \ldots, \mu_{p-1}\right),
$$

which contains one variable and $p$ parameters.

Assume that $\Phi_{s}\left(y, \mu_{0}, \mu_{1}, \ldots, \mu_{p-1}\right)$ has a nonhyperbolic fixed point at $\left(\bar{x}_{0}, \bar{\mu}\right)=\left(\bar{x}_{0}, \bar{\mu}_{0}, \bar{\mu}_{1}, \ldots\right.$, $\left.\bar{\mu}_{p-1}\right)$, i.e. $\Phi_{s}\left(\bar{x}_{0}, \bar{\mu}\right)=\bar{x}_{0}$, such that

$$
\frac{\partial \Phi_{s}}{\partial y}\left(\bar{x}_{0}, \bar{\mu}\right)=1 .
$$

Shifting this equilibrium point to the origin $0 \in \mathbb{R}^{p+1}$, it follows that the nonhyperbolicity conditions are given by

$$
\Phi_{s}(0)=0 \quad \text { and } \quad \frac{\partial \Phi_{s}}{\partial y}(0)=1
$$

The Taylor series of $\Phi_{s}(y, \boldsymbol{\mu})$ is given by

$$
\Phi_{s}(y, \boldsymbol{\mu})=\Phi_{s}(0)+\sum_{i=0}^{p-1} \frac{\partial \Phi_{s}}{\partial \mu_{i}}(0) \mu_{i}+\frac{\partial \Phi_{s}}{\partial y}(0) y+\sum_{i=0}^{p-1} \frac{\partial^{2} \Phi_{s}}{\partial \mu_{i} \partial y}(0) \mu_{i} y+\frac{1}{2} \frac{\partial^{2} \Phi_{s}}{\partial y^{2}}(0) y^{2}+O\left(y^{3}, \mu_{i}^{2}\right) .
$$

Using (2), it follows

$$
\Phi_{s}(y, \boldsymbol{\mu})=y+\sum_{i=0}^{p-1} \frac{\partial \Phi_{s}}{\partial \mu_{i}}(0) \mu_{i}+\sum_{i=0}^{p-1} \frac{\partial^{2} \Phi_{s}}{\partial \mu_{i} \partial y}(0) \mu_{i} y+\frac{1}{2} \frac{\partial^{2} \Phi_{s}}{\partial y^{2}}(0) y^{2}+O\left(y^{3}, \mu_{i}^{2}\right) .
$$

In the difference equations notation

$$
y_{n+1}=y_{n}+\sum_{i=0}^{p-1} \frac{\partial \Phi_{s}}{\partial \mu_{i}}(0) \mu_{i}+\sum_{i=0}^{p-1} \frac{\partial^{2} \Phi_{s}}{\partial \mu_{i} \partial y}(0) \mu_{i} y_{n}+\frac{1}{2} \frac{\partial^{2} \Phi_{s}}{\partial y^{2}}(0) y_{n}^{2}+O\left(y^{3}, \mu_{i}^{2}\right) .
$$

(Terms of higher order will be omitted.)

Introducing a new variable $v=y-\delta$, where $\delta$ is a parameter, it follows that $v_{n}=y_{n}-\delta$ and consequently

$$
\begin{aligned}
v_{n+1}= & v_{n}+\sum_{i=0}^{p-1} \frac{\partial \Phi_{s}}{\partial \mu_{i}}(0) \mu_{i}+\sum_{i=0}^{p-1} \frac{\partial^{2} \Phi_{s}}{\partial \mu_{i} \partial y}(0) \mu_{i}\left(\delta+v_{n}\right)+\frac{1}{2} \frac{\partial^{2} \Phi_{s}}{\partial y^{2}}(0)\left(\delta+v_{n}\right)^{2} \\
= & v_{n}+\sum_{i=0}^{p-1} \frac{\partial \Phi_{s}}{\partial \mu_{i}}(0) \mu_{i}+\sum_{i=0}^{p-1} \frac{\partial^{2} \Phi_{s}}{\partial \mu_{i} \partial y}(0) \mu_{i} \delta+\frac{1}{2} \frac{\partial^{2} \Phi_{s}}{\partial y^{2}}(0) \delta^{2}+\left(\sum_{i=0}^{p-1} \frac{\partial^{2} \Phi_{s}}{\partial \mu_{i} \partial y}(0) \mu_{i}+\frac{\partial^{2} \Phi_{s}}{\partial y^{2}}(0) \delta\right) v_{n} \\
& +\frac{1}{2} \frac{\partial^{2} \Phi_{s}}{\partial y^{2}}(0) v_{n}^{2} .
\end{aligned}
$$

To remove the linear terms $\sum_{i=0}^{p-1} \frac{\partial^{2} \Phi_{s}}{\partial \mu_{i} \partial y}(0) \mu_{i}+\frac{\partial^{2} \Phi_{s}}{\partial y^{2}}(0) \delta$, we require that

$$
\delta=-\frac{\sum_{i=0}^{p-1} \frac{\partial^{2} \Phi_{s}}{\partial \mu_{i} \partial y}(0) \mu_{i}}{\frac{\partial^{2} \Phi_{s}}{\partial y^{2}}(0)} .
$$

Obviously we need to assume that $\frac{\partial^{2} \Phi_{s}}{\partial y^{2}}(0) \neq 0$. Using this value of $\delta$, our map becomes

$$
v_{n+1}=v_{n}+\sum_{i=0}^{p-1} \frac{\partial \Phi_{s}}{\partial \mu_{i}}(0) \mu_{i}-\frac{1}{2} \frac{\left(\sum_{i=0}^{p-1} \frac{\partial^{2} \Phi_{s}}{\partial y \partial \mu_{i}}(0) \mu_{i}\right)^{2}}{\frac{\partial^{2} \Phi_{s}}{\partial y^{2}}(0)}+\frac{1}{2} \frac{\partial^{2} \Phi_{s}}{\partial y^{2}}(0) v_{n}^{2} .
$$


Now we introduce a new parameter $\beta$ such that

$$
\left\{\begin{array}{l}
\beta=\sum_{i=0}^{p-1} \frac{\partial \Phi_{s}}{\partial \mu_{i}}(0) \mu_{i}-\frac{1}{2} \frac{\left(\sum_{i=0}^{p-1} \frac{\partial^{2} \Phi_{s}}{\partial \mu_{i} \partial y}(0) \mu_{i}\right)^{2}}{\frac{\partial^{2} \Phi_{s}}{\partial y^{2}}(0)} \\
\beta=-\left(\sum_{i=0}^{p-1} \frac{\partial \Phi_{s}}{\partial \mu_{i}}(0) \mu_{i}-\frac{1}{2} \frac{\left(\sum_{i=0}^{p-1} \frac{\partial^{2} \Phi_{s}}{\partial \mu_{i} \partial y}(0) \mu_{i}\right)^{2}}{\frac{\partial^{2} \Phi_{s}}{\partial y^{2}}(0)}\right) \\
\text { if } \prod_{i=0}^{p-1} \frac{\partial \Phi_{s}}{\partial \mu_{i}}(0)>0
\end{array}\right.
$$

This assumption yields

$$
\frac{\partial \Phi_{s}}{\partial \mu_{0}}(0) \neq 0, \frac{\partial \Phi_{s}}{\partial \mu_{1}}(0) \neq 0, \ldots, \frac{\partial \Phi_{s}}{\partial \mu_{p-1}}(0) \neq 0 .
$$

Hence, the new map is now

$$
v_{n+1}=v_{n} \pm \beta+\frac{1}{2} \frac{\partial^{2} \Phi_{s}}{\partial y^{2}}(0) v_{n}^{2} .
$$

Making the changes of variable $z=\frac{1}{2}\left|\frac{\partial^{2} \Phi_{s}}{\partial y^{2}}(0)\right| v$, our equation becomes

$$
\begin{aligned}
z_{n+1} & =\frac{1}{2}\left|\frac{\partial^{2} \Phi_{s}}{\partial y^{2}}(0)\right| v_{n+1} \\
& =z_{n} \pm \beta \frac{1}{2}\left|\frac{\partial^{2} \Phi_{s}}{\partial y^{2}}(0)\right|+\frac{\frac{1}{2} \frac{\partial^{2} \Phi_{s}}{\partial y^{2}}(0)}{\frac{1}{2}\left|\frac{\partial^{2} \Phi_{s}}{\partial y^{2}}(0)\right|} \eta_{n}^{2} \\
& =z_{n} \pm \sigma \pm z_{n}^{2}
\end{aligned}
$$

where $\sigma=\beta \frac{1}{2}\left|\frac{\partial^{2} \Phi_{s}}{\partial y^{2}}(0)\right|$. Equation (4) is the normal form of the saddle-node bifurcation.

We now summarize these ideas in the following theorem.

Theorem 1. If the difference equation $y_{n+1}=$ $\Phi_{s}\left(y_{n}, \mu_{0}, \mu_{1}, \ldots, \mu_{p-1}\right)$ has a fixed point at $0 \in$ $\mathbb{R}^{p+1}$ such that

$$
\begin{aligned}
\frac{\partial \Phi_{s}}{\partial y}(0)=1, \frac{\partial \Phi_{s}}{\partial \mu_{0}}(0) \neq 0, & \\
\frac{\partial \Phi_{s}}{\partial \mu_{1}}(0) & \neq 0, \ldots, \frac{\partial \Phi_{s}}{\partial \mu_{p-1}}(0) \neq 0,
\end{aligned}
$$

and

$$
\frac{\partial^{2} \Phi_{s}}{\partial y^{2}}(0) \neq 0
$$

Then the normal forms of the map $\Phi_{s}$ in a small neighborhood of the fixed point 0 are given by

$$
z_{n+1}=z_{n} \pm \sigma \pm z_{n}^{2}
$$

and the saddle-node bifurcation takes place.

Example 4.1. Let us consider the nonautonomous one-dimensional Ricker difference equation given by

$$
x_{n+1}=x_{n} e^{\lambda_{n}-x_{n}}, \quad n \in \mathbb{Z}^{+},
$$

where $\lambda_{n}=\lambda_{n} \bmod p>0$ such that $\lambda_{n} \neq 2$, for all $n \in \mathbb{Z}^{+}$. Write Eq. (5) as $x_{n+1}=R_{n}\left(x_{n}\right)$ where $R_{n}(x)=x e^{\lambda_{n}-x}$ and let $p=2$ be its minimal period.

Since each map $R_{n}=R_{n \bmod 2}, n \in \mathbb{Z}^{+}$is one to one with respect to parameter $\lambda_{n}=\lambda_{n \bmod 2}>0$, it follows by Elaydi et al. [2011] that the only possible cycles for Eq. (5) are cycles with minimal period $r, r=2 t, t=1,2, \ldots$ Let $C_{2}=\left\{\bar{x}_{0}, \bar{x}_{1}\right\}$ be a nontrivial two-periodic cycle of Eq. (5), i.e. $\bar{x}_{0}=R_{1} \circ R_{0}\left(\bar{x}_{0}\right)$. Simplifying this equation one obtains

$$
\lambda_{0}+\lambda_{1}-\bar{x}_{0}=\bar{x}_{1}=\bar{x}_{0} e^{\lambda_{0}-\bar{x}_{0}} .
$$

Since the derivative along the periodic cycle $C_{2}$ is given by

$$
\left(1-\bar{x}_{0}\right)\left(1-\bar{x}_{1}\right),
$$

one has a bifurcation when $\left(1-\bar{x}_{0}\right)\left(1-\bar{x}_{1}\right)=1$, or equivalently

$$
\left(1-\lambda_{0}-\lambda_{1}+\bar{x}_{0}\right)\left(1-\bar{x}_{0}\right)=1 .
$$

Equation (7) has the solutions

$\bar{x}_{0}=\bar{x}_{ \pm}^{s n}=\frac{1}{2}\left(\lambda_{0}+\lambda_{1} \pm \sqrt{\left(\lambda_{0}+\lambda_{1}\right)\left(\lambda_{0}+\lambda_{1}-4\right)}\right)$. 
Since $\lambda_{0}, \lambda_{1}>0, \lambda_{0} \neq 2$ and $\lambda_{1} \neq 2$ it follows that $\bar{x}_{0} \in \mathbb{R}$ if $\lambda_{0}+\lambda_{1}>4$. Notice that the composition map is given by

$$
\Phi_{2}(x)=R_{1} \circ R_{0}(x)=x e^{\lambda_{0}+\lambda_{1}-x\left(1+e^{\lambda_{0}-x}\right)} .
$$

At the fixed point $\bar{x}_{0}$ of $\Phi_{2}$, one has $\bar{x}_{0}>0$, $\left.\frac{\partial}{\partial x}\left(\Phi_{2}(x)\right)\right|_{x=\bar{x}_{0}}=1,\left.\frac{\partial}{\partial \lambda_{1}}\left(\Phi_{2}(x)\right)\right|_{x=\bar{x}_{0}}=\bar{x}_{0}>0$ and

$$
\left.\frac{\partial}{\partial \lambda_{0}}\left(\Phi_{2}(x)\right)\right|_{x=\bar{x}_{0}}=\left(1-\lambda_{0}-\lambda_{1}+\bar{x}_{0}\right) \bar{x}_{0} .
$$

A straightforward computation results that when $\bar{x}_{0}=\bar{x}_{+}^{s n}$ we have

$$
\begin{aligned}
\frac{\partial}{\partial \lambda_{0}} & \left.\left(\Phi_{2}(x)\right)\right|_{x=\bar{x}_{0}} \\
& =\bar{x}_{0} \frac{2-\lambda_{0}-\lambda_{1}+\sqrt{\left(\lambda_{0}+\lambda_{1}\right)\left(\lambda_{0}+\lambda_{1}-4\right)}}{2} \\
& <0,
\end{aligned}
$$

since

$$
\sqrt{\left(\lambda_{0}+\lambda_{1}\right)\left(\lambda_{0}+\lambda_{1}-4\right)}<\lambda_{0}+\lambda_{1}-2 .
$$

Similarly, when $\bar{x}_{0}=\bar{x}_{-}^{s n}$ we have

$$
\left.\frac{\partial}{\partial \lambda_{0}}\left(\Phi_{2}(x)\right)\right|_{x=\bar{x}_{0}}<0 \text {. }
$$

Consequently, $\left.\frac{\partial}{\partial \lambda_{0}}\left(\Phi_{2}(x)\right)\right|_{x=\bar{x}_{0}} \neq 0$.

Substituting the values of the bifurcation solutions in (6) yields two equations of the form

$$
\lambda_{0}+\lambda_{1}-y=y e^{\lambda_{0}-y},
$$

where $y$ is given by $\bar{x}_{+}^{s n}$ or $\bar{x}_{-}^{s n}$.

The implicit function theorem (see for instance [Protter \& Morrey, 1991; Zeidler, 1993]) guarantees the existence of solutions of the two equations given in (9). The exact solutions of these two equations are represented implicitly in the parameter space $\left(\lambda_{0}, \lambda_{1}\right)$ in Fig. 1 (gray curves). In Sec. 7 we will give more details about the regions represented in this figure.

Hence, from the implicit solutions, it is enough to consider the values of the parameters in the interval $(2,3)$ when the derivative equals 1 . Since

$$
\begin{aligned}
\frac{\partial^{2}}{\partial x^{2}}\left(\Phi_{2}(x)\right) & \left.\right|_{x=\bar{x}_{0}} \\
= & -1-e^{\lambda_{0}-\bar{x}_{0}}+\left(3-\bar{x}_{0}\right)\left(\lambda_{0}+\lambda_{1}-\bar{x}_{0}\right) \\
& +\left(1-\bar{x}_{0}\right)\left(1-\lambda_{0}-\lambda_{1}+\bar{x}_{0}\right)\left(-1-e^{\lambda_{0}-\bar{x}_{0}}\right. \\
& \left.+\lambda_{0}+\lambda_{1}-\bar{x}_{0}\right),
\end{aligned}
$$

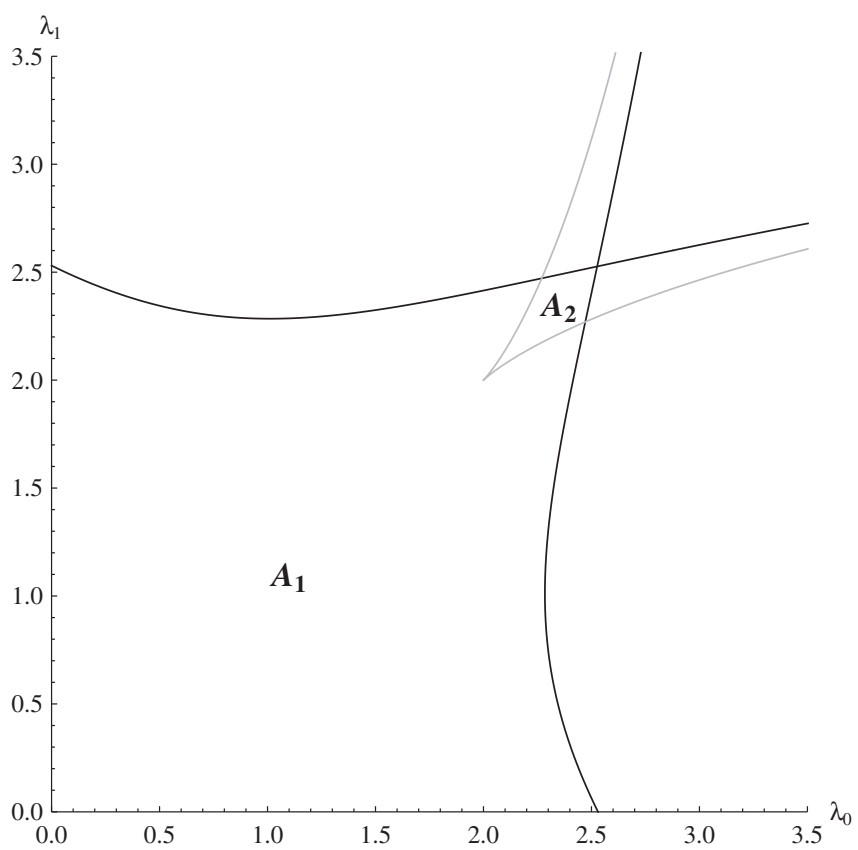

Fig. 1. The bifurcation curves of a two-periodic cycle, in the parameter space, of the two-periodic Ricker equation.

it follows that

$$
\begin{aligned}
& \left.\frac{\partial^{2}}{\partial x^{2}}\left(\Phi_{2}(x)\right)\right|_{x=\bar{x}_{+}^{s n}} \\
& \quad<0 \text { for all } \lambda_{0}, \lambda_{1} \in(2,3) \times(2,3)
\end{aligned}
$$

and

$$
\begin{aligned}
& \left.\frac{\partial^{2}}{\partial x^{2}}\left(\Phi_{2}(x)\right)\right|_{x=\bar{x}_{-}^{s n}} \\
& \quad>0 \text { for all } \lambda_{0}, \lambda_{1} \in(2,3) \times(2,3) .
\end{aligned}
$$

Since the conditions in Theorem 1 are satisfied, it follows that on these bifurcation curves occurs a saddle-node bifurcation.

\section{Transcritical Bifurcation}

Assume that the map $\Phi_{s}\left(y, \mu_{0}, \mu_{1}, \ldots, \mu_{p-1}\right)$ has a nonhyperbolic fixed point at $0 \in \mathbb{R}^{p+1}$ (after shifting the fixed point $\left(\bar{x}_{0}, \bar{\mu}_{0}, \bar{\mu}_{1}, \ldots, \bar{\mu}_{p-1}\right)$ to the origin) such that

$$
\begin{aligned}
\Phi_{s}(0) & =0 \quad \text { and } \quad \frac{\partial \Phi_{s}}{\partial y}(0)=1 \\
\frac{\partial \Phi_{s}}{\partial \mu_{0}}(0) & =0, \frac{\partial \Phi_{s}}{\partial \mu_{1}}(0)=0, \ldots, \frac{\partial \Phi_{s}}{\partial \mu_{p-1}}(0)=0
\end{aligned}
$$


and

$$
\frac{\partial^{2} \Phi_{s}}{\partial \mu_{i} \partial \mu_{j}}(0)=0, \quad \text { for all } i, j \in\{0,1, \ldots, p-1\} \text {. }
$$

Let us write the vector parameter $\left(\mu_{0}, \mu_{1}, \ldots\right.$, $\left.\mu_{p-1}\right)$ as $\boldsymbol{\mu}$ and write the equation as

$$
y_{n+1}=\Phi_{s}\left(y_{n}, \boldsymbol{\mu}\right) .
$$

The Taylor series of (13) is given by

$$
\begin{aligned}
\Phi_{s}(y, \boldsymbol{\mu})= & \Phi_{s}(0)+\sum_{i=0}^{p-1} \frac{\partial \Phi_{s}}{\partial \mu_{i}}(0) \mu_{i}+\frac{\partial \Phi_{s}}{\partial y}(0) y \\
& +\sum_{i=0}^{p-1} \frac{\partial^{2} \Phi_{s}}{\partial \mu_{i} \partial y}(0) \mu_{i} y+\frac{1}{2} \sum_{i=0}^{p-1} \frac{\partial^{2} \Phi_{s}}{\partial \mu_{i}^{2}}(0) \mu_{i}^{2} \\
& +\sum_{i=0}^{p-1} \sum_{j=i+1}^{p-1} \frac{\partial^{2} \Phi_{s}}{\partial \mu_{i} \mu_{j}}(0) \mu_{i} \mu_{j} \\
& +\frac{1}{2} \frac{\partial^{2} \Phi_{s}}{\partial y^{2}}(0) y^{2}+O\left(y^{3}, \mu_{i}^{3}\right) .
\end{aligned}
$$

Using (10)-(12), the Taylor series is now given by

$$
\begin{aligned}
\Phi_{s}(y, \boldsymbol{\mu})= & y+\sum_{i=0}^{p-1} \frac{\partial^{2} \Phi_{s}}{\partial \mu_{i} \partial y}(0) \mu_{i} y \\
& +\frac{1}{2} \frac{\partial^{2} \Phi_{s}}{\partial y^{2}}(0) y^{2}+O\left(y^{3}, \mu_{i}^{3}\right) .
\end{aligned}
$$

Introducing a new parameter $\beta$ such that

$$
\left\{\begin{aligned}
\beta= & \sum_{i=0}^{p-1} \frac{\partial^{2} \Phi_{s}}{\partial \mu_{i} \partial y}(0) \mu_{i} \\
& +O\left(y^{2}, \mu_{i}^{3}\right), \quad \text { if } \prod_{i=0}^{p-1} \frac{\partial^{2} \Phi_{s}}{\partial \mu_{i} \partial y}(0)>0 \\
\beta= & -\sum_{i=0}^{p-1} \frac{\partial^{2} \Phi_{s}}{\partial \mu_{i} \partial y}(0) \mu_{i} \\
& +O\left(y^{2}, \mu_{i}^{3}\right), \quad \text { if } \prod_{i=0}^{p-1} \frac{\partial^{2} \Phi_{s}}{\partial \mu_{i} \partial y}(0)<0
\end{aligned}\right.
$$

we notice that locally, i.e. in an appropriated neighborhood of the solution of the above bifurcation conditions (which was shifted to the origin), the value of $\beta$ is dominated by $\sum_{i=0}^{p-1} \frac{\partial^{2} \Phi_{s}}{\partial \mu_{i} \partial y}(0) \mu_{i}$ which is different from zero (we treat this case as usual, see [Arrowsmith \& Place, 1990, p. 219] for instance). Finally, we rescale the Taylor series using $\frac{1}{2}\left|\frac{\partial^{2} \Phi_{s}}{\partial y^{2}}(0)\right| y=v$ to get the normal form

$$
v_{n+1}=(1 \pm \beta) v_{n} \pm v_{n}^{2} .
$$

The genericity conditions for normalization are

$$
\begin{gathered}
\frac{\partial^{2} \Phi_{s}}{\partial y^{2}}(0) \neq 0, \frac{\partial^{2} \Phi_{s}}{\partial \mu_{0} \partial y}(0) \neq 0, \frac{\partial^{2} \Phi_{s}}{\partial \mu_{1} \partial y}(0) \neq 0, \ldots, \\
\frac{\partial^{2} \Phi_{s}}{\partial \mu_{p-1} \partial y}(0) \neq 0 .
\end{gathered}
$$

Theorem 2. Let $0 \in \mathbb{R}^{p+1}$ be a nonhyperbolic fixed point of the difference equation

$$
y_{n+1}=\Phi_{s}\left(y_{n}, \boldsymbol{\mu}\right)
$$

such that $\Phi_{s}(0)=0, \frac{\partial \Phi_{s}}{\partial y}(0)=1, \frac{\partial \Phi_{s}}{\partial \mu_{i}}(0)=0$ for all $i \in\{0,1, \ldots, p-1\}$, and

$$
\frac{\partial^{2} \Phi_{s}}{\partial \mu_{i} \partial \mu_{j}}(0)=0, \quad \text { for all } i, j \in\{0,1, \ldots, p-1\} \text {. }
$$

If

$$
\frac{\partial^{2} \Phi_{s}}{\partial \mu_{i} \partial y}(0) \neq 0 \quad \text { for all } i \in\{0,1, \ldots, p-1\},
$$

then the normal forms of the map $\Phi_{s}$ in a small neighborhood of the fixed point 0 are given by

$$
v_{n+1}=(1 \pm \beta) v_{n} \pm v_{n}^{2},
$$

and the transcritical bifurcation takes place.

Example 5.1. Consider the difference equation

$$
x_{n+1}=x_{n}\left(a_{n}-x_{n}\right)\left(a_{n+1}-x_{n}^{2}\left(a_{n}-x_{n}\right)\right),
$$

with $a_{n}>0$ for all $n=0,1,2, \ldots$ Let $a_{n}=a_{n \bmod 2 \text {, }}$ for all $n=0,1,2, \ldots$. This leads to a two-periodic difference equation. The composition operator $\Phi$ is given by

$$
\Phi(x)=x\left(a_{0}-x\right)\left(a_{1}-x^{2}\left(a_{0}-x\right)\right) .
$$

Consider the zero fixed point of $\Phi$. This fixed point is locally asymptotically stable when $a_{0} a_{1}<1$. Let $a_{0} a_{1}=1$. A straightforward computation results that $\frac{\partial \Phi}{\partial x}\left(0, a_{0}, a_{1}\right)=1$. Moreover,

$$
\begin{aligned}
\frac{\partial \Phi}{\partial a_{0}}\left(0, a_{0}, a_{1}\right) & =\frac{\partial \Phi}{\partial a_{1}}\left(0, a_{0}, a_{1}\right) \\
& =\frac{\partial^{2} \Phi}{\partial a_{0}^{2}}\left(0, a_{0}, a_{1}\right)
\end{aligned}
$$




$$
\begin{aligned}
& =\frac{\partial^{2} \Phi}{\partial a_{1}^{2}}\left(0, a_{0}, a_{1}\right) \\
& =\frac{\partial^{2} \Phi}{\partial a_{0} \partial a_{1}}\left(0, a_{0}, a_{1}\right) \\
& =0 .
\end{aligned}
$$

Furthermore,

$$
\begin{aligned}
\frac{\partial^{2} \Phi}{\partial x^{2}}\left(0, a_{0}, a_{1}\right) & =-2 a_{1}<0 \\
\frac{\partial^{2} \Phi}{\partial a_{0} \partial x}\left(0, a_{0}, a_{1}\right) & =a_{1}>0
\end{aligned}
$$

and

$$
\frac{\partial^{2} \Phi}{\partial a_{1} \partial x}\left(0, a_{0}, a_{1}\right)=a_{0}>0 .
$$

Hence the required conditions for the transcritical bifurcation are satisfied. Thus on the curve $a_{0} a_{1}=1$ an exchange of stability takes place.

Example 5.2. Consider the nonautonomous $p$ periodic logistic equation given by $x_{n+1}=f_{n}\left(x_{n}\right)$, $n=0,1,2, \ldots$, where the sequence of maps are given by $f_{n}(x)=\mu_{n} x(1-x), \mu_{n} \in(0,4)$, for all $n=0,1,2, \ldots$.

The derivative of composition operator is given by

$$
\Phi_{p}^{\prime}(x)=\prod_{i=0}^{p-1} \mu_{i}\left(1-2 \Phi_{i}(x)\right) .
$$

At the fixed point 0 we have $\Phi_{p}^{\prime}(0)=\prod_{i=0}^{p-1} \mu_{i}$. Hence, the fixed point 0 is locally asymptotically stable when $\prod_{i=0}^{p-1} \mu_{i}<1$. In order to have bifurcation we need to assume that

$$
\prod_{i=0}^{p-1} \mu_{i}=1
$$

A straightforward computation shows that

$$
\begin{aligned}
& \left.\frac{\partial}{\partial \mu_{i}}\left(\Phi_{p}(x)\right)\right|_{x=0}=0, \quad \text { for all } i=0,1,2, \ldots, p-1, \\
& \left.\frac{\partial^{2}}{\partial \mu_{i} \partial \mu_{j}}\left(\Phi_{p}(x)\right)\right|_{x=0} \\
& \quad=0, \quad \text { for all } i, j \in\{0,1,2, \ldots, p-1\}, \\
& \left.\frac{\partial^{2}}{\partial \mu_{i} \partial x}\left(\Phi_{p}(x)\right)\right|_{x=0}=\prod_{j=0, j \neq i}^{p-1} \mu_{j}=\frac{1}{\mu_{i}} \neq 0,
\end{aligned}
$$$$
\text { for all } i=0,1,2, \ldots, p-1 \text {, }
$$

and

$$
\begin{aligned}
\Phi_{p}^{\prime \prime}(0) & =-2 \prod_{i=0}^{p-1} \mu_{i}\left(1+\sum_{i=0}^{p-2} \prod_{j=0}^{i} \mu_{j}\right) \\
& =-2\left(1+\sum_{i=0}^{p-2} \prod_{j=0}^{i} \mu_{j}\right) \neq 0 .
\end{aligned}
$$

Hence, the fixed point 0 of the $p$-periodic logistic equation undergoes a transcritical bifurcation when $\prod_{i=0}^{p-1} \mu_{i}=1$.

\section{Pitchfork Bifurcation}

Suppose that the map $y_{n+1}=\Phi_{s}\left(y_{n}, \boldsymbol{\mu}\right)$ has a nonhyperbolic fixed point at $0 \in \mathbb{R}^{p+1}$ (after shifting the fixed point $\left(\bar{x}_{0}, \bar{\mu}_{0}, \bar{\mu}_{1}, \ldots, \bar{\mu}_{p-1}\right)$ to the origin) such that

$$
\Phi_{s}(0)=0 \quad \text { and } \quad \frac{\partial \Phi_{s}}{\partial y}(0)=1
$$

and

$$
\frac{\partial \Phi}{\partial \mu_{0}}(0)=\frac{\partial \Phi}{\partial \mu_{1}}(0)=\cdots=\frac{\partial \Phi}{\partial \mu_{p-1}}(0)=\frac{\partial^{2} \Phi}{\partial y^{2}}=0 .
$$

Hence, the Taylor series is given by

$$
\begin{aligned}
\Phi(y, \boldsymbol{\mu})= & y+\sum_{i=0}^{p-1} \frac{\partial^{2} \Phi_{s}}{\partial \mu_{i} \partial y}(0) \mu_{i} y \\
& +\frac{1}{6} \frac{\partial^{3} \Phi_{s}}{\partial y^{3}}(0) y^{3} .
\end{aligned}
$$

Let $\beta$ be a new parameter such that

$$
\begin{cases}\beta=\sum_{i=0}^{p-1} \frac{\partial^{2} \Phi_{s}}{\partial \mu_{i} \partial y}(0) \mu_{i} & \text { if } \prod_{i=0}^{p-1} \frac{\partial^{2} \Phi_{s}}{\partial \mu_{i} \partial y}>0 \\ \beta=-\sum_{i=0}^{p-1} \frac{\partial^{2} \Phi_{s}}{\partial \mu_{i} \partial y}(0) \mu_{i} & \text { if } \prod_{i=0}^{p-1} \frac{\partial^{2} \Phi_{s}}{\partial \mu_{i} \partial y}<0\end{cases}
$$

Hence, the equation now becomes

$$
y_{n+1}=y_{n} \pm \beta y_{n}+\frac{1}{6} \frac{\partial^{3} \Phi_{s}}{\partial y^{3}}(0) y_{n}^{3}
$$

and by the change of variable $v=\sqrt{\frac{1}{6}\left|\frac{\partial^{3} \Phi_{s}}{\partial y^{3}}(0)\right|} y$ we obtain

$$
v_{n+1}=v_{n} \pm \beta v_{n} \pm v_{n}^{3} .
$$


Theorem 3. Consider that the map $y_{n+1}=\Phi_{s}\left(y_{n}\right.$, $\boldsymbol{\mu})$ has a nonhyperbolic fixed point at 0 such that

$$
\Phi_{s}(0)=0 \quad \text { and } \quad \frac{\partial \Phi_{s}}{\partial y}(0)=1
$$

and

$\frac{\partial \Phi}{\partial \mu_{0}}(0)=\frac{\partial \Phi}{\partial \mu_{1}}(0)=\cdots=\frac{\partial \Phi}{\partial \mu_{p-1}}(0)=\frac{\partial^{2} \Phi}{\partial y^{2}}(0)=0$

If

$\frac{\partial^{2} \Phi_{s}}{\partial \mu_{0} \partial y}(0) \neq 0, \frac{\partial^{2} \Phi_{s}}{\partial \mu_{1} \partial y}(0) \neq 0, \ldots, \frac{\partial^{2} \Phi_{s}}{\partial \mu_{p-1} \partial y}(0) \neq 0$

and

$$
\frac{\partial^{3} \Phi_{s}}{\partial y^{3}}(0) \neq 0
$$

then the normal forms of the map $\Phi_{s}$ in a small neighborhood of the fixed point 0 are given by

$$
v_{n+1}=v_{n} \pm \beta v_{n} \pm v_{n}^{3},
$$

and the pitchfork bifurcation takes place.

Example 6.1. Consider the difference equation $x_{n+1}=f_{n}\left(x_{n}\right)$ where $f_{n}(x)=\mu_{n} x-x^{3}, \mu_{n}>0$ for all $n=0,1,2, \ldots$ Let $\mu_{n}=\mu_{n} \bmod p$, for all $n=0,1,2, \ldots$ This leads to a $p$-periodic difference equation since $f_{n}=f_{n+p}$, for all $n=0,1,2, \ldots$. The composition operator is given by

$$
\Phi_{p}(x)=f_{p-1} \circ f_{p-2} \circ \cdots \circ f_{0}(x) .
$$

The derivative of the composition operator is

$$
\Phi_{p}^{\prime}(x)=\prod_{i=0}^{p-1}\left(\mu_{i}-3\left(\Phi_{i}(x)\right)^{2}\right) .
$$

Hence, the fixed point $x^{*}=0$ is locally asymptotically stable when $\prod_{i=0}^{p-1} \mu_{i}<1$ since

$$
\Phi_{p}^{\prime}(0)=\prod_{i=0}^{p-1} \mu_{i}
$$

When $\prod_{i=0}^{p-1} \mu_{i}=1$, a bifurcation takes place. Computation shows that

$$
\frac{\partial \Phi_{p}}{\partial \mu_{i}}(0)=0, \quad \text { for all } i=0,1, \ldots, p-1 .
$$

A straightforward computation results that when $\prod_{i=0}^{p-1} \mu_{i}=1$ we have

$$
\frac{\partial^{2} \Phi_{p}}{\partial \mu_{i} \partial x}(0)=\frac{1}{\mu_{i}}>0, \quad \text { for all } i=0, \ldots, p-1 .
$$

Moreover,

$$
\Phi_{p}^{\prime \prime}(x)=-6 \sum_{j=0}^{p-1} \Phi_{j}(x) \Phi_{j}^{\prime}(x) \prod_{i=0, i \neq j}^{p-1}\left(\mu_{i}-3 \Phi_{i}^{2}\right) .
$$

Hence, $\Phi_{p}^{\prime \prime}(0)=0$.

The third derivative of the composition operator is given by

$$
\begin{aligned}
\Phi_{p}^{\prime \prime \prime}(x)= & -6 \sum_{k=0}^{p-1}\left(\Phi_{k}^{\prime \prime}(x) \Phi_{k}(x) \prod_{i=0, i \neq k}^{p-1}\left(\mu_{i}-3 \Phi_{i}^{2}\right)\right. \\
& +\left(\Phi_{k}^{\prime}(x)\right)^{2} \prod_{i=0, i \neq k}^{p-1}\left(\mu_{i}-3 \Phi_{i}^{2}\right) \\
& +\Phi_{k}^{\prime}(x) \Phi_{k}(x)\left(-6 \sum_{j=0, j \neq k}^{p-1} \Phi_{k}^{\prime}(x) \Phi_{k}(x)\right. \\
& \left.\left.\times \prod_{i=0, i \neq j, i \neq k}^{p-1}\left(\mu_{i}-3 \Phi_{i}^{2}\right)\right)\right) .
\end{aligned}
$$

Consequently,

$$
\Phi_{p}^{\prime \prime \prime}(0)=-6 \sum_{k=0}^{p-1} \frac{1}{\mu_{k}}\left(\prod_{i=0}^{k-1} \mu_{i}\right)^{2} \neq 0 .
$$

Hence, at $\prod_{i=0}^{p-1} \mu_{i}=1$, the pitchfork bifurcation takes place. (Notice that it is a supercritical bifurcation.) In Fig. 2, the bifurcation surface is presented in the parameter space $\left(\mu_{0}, \mu_{1}, \mu_{2}\right)$ when $p=3$.

Example 6.2. Consider the difference equation $x_{n+1}=H_{n}\left(x_{n}\right)$ where $H_{n}(x)=\mu_{n} \sin (x)$, and $\mu_{n}>0$, for all $n=0,1,2, \ldots$ Let $\mu_{n}=\mu_{n} \bmod p$, for all $n=0,1,2, \ldots$ Hence, we have $H_{n+p}=H_{n}$ for all $n=0,1,2, \ldots$.

The composition operator is given by

$$
\begin{aligned}
& \Phi_{p}(x) \\
& \quad=\mu_{p-1} \sin \left(\mu_{p-2} \sin \left(\cdots\left(\mu_{1} \sin \left(\mu_{0} \sin (x)\right)\right) \cdots\right)\right) .
\end{aligned}
$$

A straightforward computation shows that

$$
\Phi_{p}^{\prime}(x)=\prod_{i=0}^{p-1} \mu_{i} \cos \left(\Phi_{i}(x)\right),
$$

and thus $\Phi_{p}^{\prime}(0)=\prod_{i=0}^{p-1} \mu_{i}$. Consequently, the fixed point $x^{*}=0$ is locally asymptotically stable when $\prod_{i=0}^{p-1} \mu_{i}<1$. Hence, we will have bifurcation when $\prod_{i=0}^{p-1} \mu_{i}=1$. 


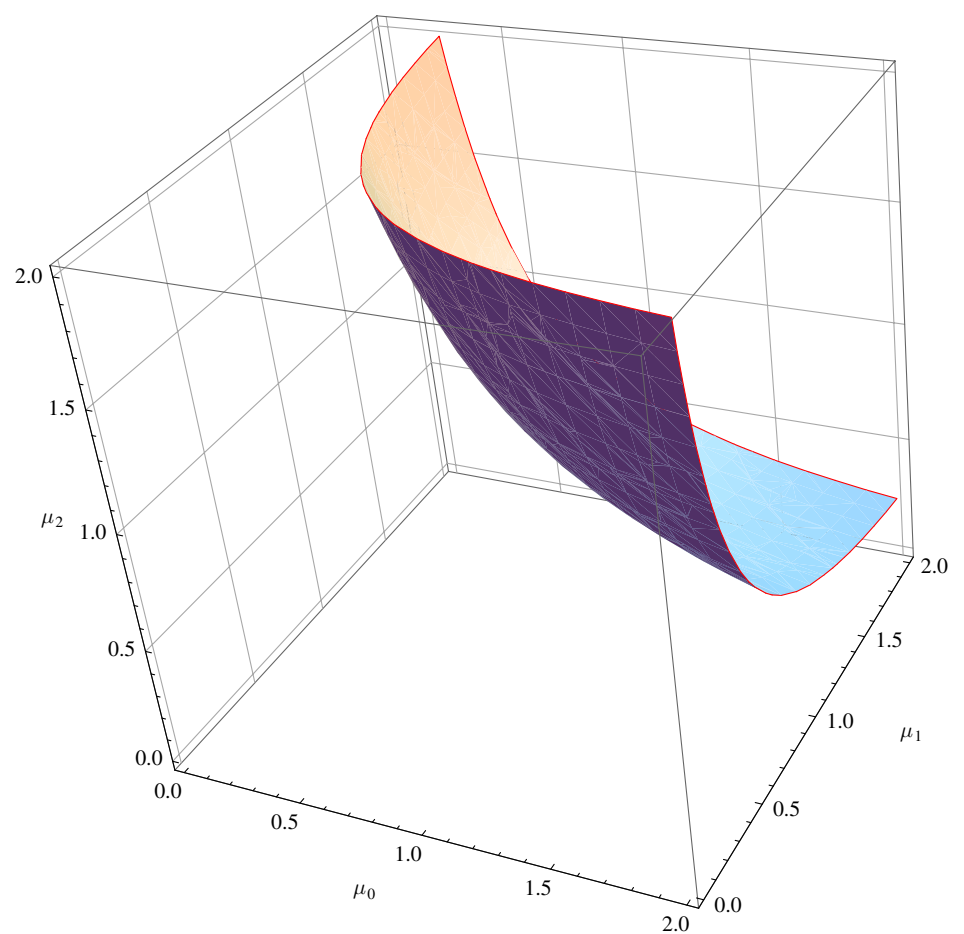

Fig. 2. The bifurcation surface where the pitchfork bifurcation of the fixed point $x^{*}=0$ of the equation $x_{n+1}=f_{n}\left(x_{n}\right)$ occurs, $f_{n}(x)=\mu_{n} x-x^{3}, \mu_{n}>0$ and $f_{n}=f_{n+3}$ for all $n=0,1,2, \ldots$

A direct computation shows that

$$
\begin{aligned}
\frac{\partial \Phi_{p}}{\partial \mu_{p-1}}(x) & =\sin \left(\Phi_{p}(x)\right) \\
\frac{\partial \Phi_{p}}{\partial \mu_{p-2}}(x) & =\sin \left(\Phi_{p-2}(x)\right) \mu_{p-1} \cos \left(\Phi_{p-1}(x)\right) \\
\frac{\partial \Phi_{p}}{\partial \mu_{p-3}}(x) & =\sin \left(\Phi_{p-3}(x)\right) \prod_{i=1}^{2} \mu_{p-i} \cos \left(\Phi_{p-i}(x)\right) \\
& \vdots \\
\frac{\partial \Phi_{p}}{\partial \mu_{0}}(x) & =\sin \left(\Phi_{0}(x)\right) \prod_{i=1}^{p-1} \mu_{p-i} \cos \left(\Phi_{p-i}(x)\right) .
\end{aligned}
$$

Hence, at the fixed point 0 we have $\frac{\partial \Phi_{p}}{\mu_{i}}(0)=0$, for all $i=0,1, \ldots, p-1$.

It can be shown that $\frac{\partial^{2} \Phi_{p}}{\partial \mu_{i} \partial x}(0)=\prod_{j=0, j \neq i}^{p-1} \mu_{j}=$ $\frac{1}{\mu_{i}}$, for all $i=0,1, \ldots, p-1$. (We omit the computations here since they are long.) Moreover,

$$
\begin{aligned}
\Phi_{p}^{\prime \prime}(x)= & -\sum_{i=0}^{p-1} \mu_{i} \Phi_{p}^{\prime}(x) \sin \left(\Phi_{i}(x)\right) \\
& \times \prod_{j=0, j \neq i}^{p-1} \mu_{j} \cos \left(\Phi_{j}(x)\right) .
\end{aligned}
$$

Hence, $\Phi_{p}^{\prime \prime}(0)=0$. From relation (18) it follows that

$$
\begin{aligned}
\Phi_{p}^{\prime \prime \prime}(x)= & -\prod_{k=0}^{p-1} \mu_{k} \sum_{i=0}^{p-1}\left(\left(\Phi_{i}^{\prime \prime}(x) \sin \left(\Phi_{i}(x)\right)\right.\right. \\
& \left.+\left(\Phi_{i}^{\prime}(x)\right)^{2} \cos \left(\Phi_{i}(x)\right)\right) \prod_{j=0, j \neq i}^{p-1} \cos \left(\Phi_{j}(x)\right) \\
& +\Phi_{i}^{\prime}(x) \sin \left(\Phi_{i}(x)\right) \frac{\partial}{\partial x} \\
& \left.\times\left(\prod_{j=0, j \neq i}^{p-1} \cos \left(\Phi_{j}(x)\right)\right)\right) .
\end{aligned}
$$

It follows that

$$
\begin{aligned}
\Phi_{p}^{\prime \prime \prime}(0) & =-\prod_{k=0}^{p-1} \mu_{k} \sum_{i=0}^{p-1}\left(\Phi_{i}^{\prime}(0)\right)^{2} \\
& =-\sum_{i=0}^{p-1}\left(\prod_{j=0}^{i-1} \mu_{j}\right) \\
& \neq 0 .
\end{aligned}
$$

Consequently, pitchfork bifurcation (supercritical) takes place at fixed point 0 when $\prod_{i=0}^{p-1} \mu_{i}=1$. 
We end this section presenting two remarks:

(1) In Example 4.1 we studied the bifurcation of a two-periodic cycle of the Ricker model when $\lambda_{i}>2, i=0,1$. If $\lambda=\lambda_{0}=\lambda_{1}$ the map representing the equation is autonomous. In particular, when $\lambda_{0}=\lambda_{1}=2$, the fixed point is given by $x^{*}=2$ and one can show that, for the composition map

$$
\Phi_{2}(x)=x e^{2 \lambda-x-x e^{\lambda-x}}
$$

we have $\Phi_{2}^{\prime}(0)=1, \frac{\partial \Phi_{2}}{\partial \lambda}(0)=0, \Phi_{2}^{\prime \prime}(0)=0$, $\frac{\partial^{2} \Phi_{2}}{\partial \lambda \partial x}(0)=2$ and $\Phi_{2}^{\prime \prime \prime}(0)=-2$. Hence, when $\lambda_{0}=\lambda_{1}=2$ the fixed point of the Ricker map undergoes a pitchfork bifurcation. This point, in the parameter space, is the vertex of the cusp represented in Fig. 1.

(2) Similar conclusion can be taken in the case of the logistic equation given in Example 5.2 when $\mu_{0}=\mu_{1}=3$. For more details about pitchfork bifurcation in two-periodic systems see [D'Aniello \& Oliveira, 2009].

\section{Period-Doubling Bifurcation}

Let us assume that the map $y_{n+1}=\Phi_{s}\left(y_{n}, \mu_{0}\right.$, $\left.\mu_{1}, \ldots, \mu_{p-1}\right)$ has a nonhyperbolic fixed point such that

$$
\begin{aligned}
\Phi_{s}\left(\bar{x}_{0}, \bar{\mu}_{0}, \bar{\mu}_{1}, \ldots, \bar{\mu}_{p-1}\right) & =\bar{x}_{0} \quad \text { and } \\
\frac{\partial \Phi_{s}}{\partial y}\left(\bar{x}_{0}, \bar{\mu}_{0}, \bar{\mu}_{1}, \ldots, \bar{\mu}_{p-1}\right) & =-1 .
\end{aligned}
$$

By shifting this equilibrium point to the origin $0 \in \mathbb{R}^{p+1}$, it follows that the nonhyperbolicity conditions becomes

$$
\Phi_{s}(0)=0 \quad \text { and } \quad \frac{\partial \Phi_{s}}{\partial y}(0)=-1 .
$$

Because the slope of $\Phi_{s}$ is -1 the fixed point 0 must exist for all $\left(\mu_{0}, \mu_{1}, \ldots, \mu_{p-1}\right)$ close to $(0$, $0, \ldots, 0)$. In fact, by the relation

$$
\Phi_{s}\left(y, \mu_{0}, \mu_{1}, \ldots, \mu_{p-1}\right)=y,
$$

it follows that

$$
\Phi_{s}(0)+\frac{\partial \Phi_{s}}{\partial y}(0) y+\sum_{i=0}^{p-1} \frac{\partial \Phi_{s}}{\partial \mu_{i}}(0) \mu_{i}=y,
$$

and consequently

$$
y=\frac{1}{2} \sum_{i=0}^{p-1} \frac{\partial \Phi_{s}}{\partial \mu_{i}}(0) \mu_{i} .
$$

This implies that we have a line of fixed points $y=Y\left(\mu_{0}, \mu_{1}, \ldots, \mu_{p-1}\right)$. Notice that we cannot use this technique close to the other nonhyperbolic fixed point where $\frac{\partial \Phi_{s}}{\partial y}(0)=1$, because in that situation the variable $y$ vanishes in Eq. (20) and we will not find any solution.

By the change of variables $z=y-Y$ we find a new map

$$
z_{n+1}=\Psi\left(z_{n}, \boldsymbol{\mu}\right)
$$

which has fixed points at $z=0$ for all $\mu_{0}, \mu_{1}, \ldots$, $\mu_{p-1}$, i.e. $\Psi\left(0, \mu_{0}, \mu_{1}, \ldots, \mu_{p-1}\right)=0$.

Therefore we now assume, without loss of generality, that we have a map $z_{n+1}=\Psi\left(z_{n}, \boldsymbol{\mu}\right)$ with the following properties

$$
\Psi(0, \boldsymbol{\mu})=0 \quad \text { and } \quad \frac{\partial \Psi}{\partial z}(0)=-1 .
$$

The Taylor expansion of $\Psi$ is given by

$$
\begin{aligned}
\Psi(z, \boldsymbol{\mu})= & \Psi(0, \boldsymbol{\mu})+\frac{\partial \Psi}{\partial z}(0, \boldsymbol{\mu}) z+\frac{\partial^{2} \Psi}{\partial z^{2}}(0, \boldsymbol{\mu}) \frac{z^{2}}{2} \\
& +\frac{\partial^{3} \Psi}{\partial z^{3}}(0, \boldsymbol{\mu}) \frac{z^{3}}{3 !}+\cdots \\
\approx & A_{1}(\boldsymbol{\mu}) z+A_{2}(\boldsymbol{\mu}) z^{2}+A_{3}(\boldsymbol{\mu}) z^{3},
\end{aligned}
$$

where

$$
\begin{gathered}
A_{1}(\boldsymbol{\mu})=\frac{\partial \Psi}{\partial z}(0, \boldsymbol{\mu}), \quad A_{2}(\boldsymbol{\mu})=\frac{1}{2} \frac{\partial^{2} \Psi}{\partial z^{2}}(0, \boldsymbol{\mu}) \text { and } \\
A_{3}(\boldsymbol{\mu})=\frac{1}{6} \frac{\partial^{3} \Psi}{\partial z^{3}}(0, \boldsymbol{\mu}) .
\end{gathered}
$$

Because it is convenient to remove the quadratic terms we use the quadratic change of variable

$$
z=u+\alpha u^{2}
$$

The inverse change of variables is given by

$$
u=\frac{-1 \pm \sqrt{1+4 \alpha z}}{2 \alpha} .
$$

Notice that the graph of (24) is a parabola in the $(u, z)$-plane open upwards with two roots $u=0$ and $u=-\alpha$. Hence we chose to work with the right branch of this parabola, because it passes through the point $z=0$ and $u=0$. Therefore, it maps our equilibrium point $z=0$ to the point $u=0$. This branch corresponds to the sign + in (25) and 
therefore the inverse change of variables is

$$
u=\frac{-1+\sqrt{1+4 \alpha z}}{2 \alpha} .
$$

Since

$$
\sqrt{1+4 \alpha z}=1+2 \alpha z-2 \alpha^{2} z^{2}+4 \alpha^{3} z^{3}+\cdots,
$$

it follows that

$$
u \approx z-\alpha z^{2}+2 \alpha^{2} z^{3} .
$$

So, in the difference equation notation we have

$$
u_{n+1}=z_{n+1}-\alpha z_{n+1}^{2}+2 \alpha^{2} z_{n}^{3} .
$$

Using (23) and removing the terms up to the third order yields

$$
\begin{aligned}
u_{n+1}= & A_{1} z_{n}+\left(A_{2}-\alpha A_{1}^{2}\right) z_{n}^{2} \\
& +\left(A_{3}-2 \alpha A_{1} A_{2}+2 \alpha^{2} A_{1}^{3}\right) z_{n}^{3} .
\end{aligned}
$$

By (24) it follows that $z^{2} \approx u^{2}+2 \alpha \mu^{3}$ and $z^{3} \approx \mu^{3}$. Hence

$$
\begin{aligned}
u_{n+1}= & A_{1} u_{n}+\left(\alpha A_{1}+A_{2}-\alpha A_{1}^{2}\right) u_{n}^{2} \\
& +\left(2 \alpha A_{2}-2 \alpha^{2} A_{1}^{2}+A_{3}-2 \alpha A_{1} A_{2}\right. \\
& \left.+2 \alpha^{2} A_{1}^{3}\right) u_{n}^{3} .
\end{aligned}
$$

Now we can remove the quadratic term and it follows that

$$
\alpha=\frac{A_{2}(\boldsymbol{\mu})}{A_{1}^{2}(\boldsymbol{\mu})-A_{1}(\boldsymbol{\mu})} .
$$

At the point $\boldsymbol{\mu}=0$ we know that $A_{1}(0)=-1$ and thus $\alpha=A_{2}(0) / 2$. Consequently, at the origin the coefficient of $u^{3}$ is now

$$
A=A_{3}+A_{2}^{2}=\frac{1}{6} \frac{\partial^{3} \Psi}{\partial z^{3}}(0)+\frac{1}{4}\left(\frac{\partial^{2} \Psi}{\partial z^{2}}(0)\right)^{2} .
$$

Hence, the new map is now

$$
u_{n+1}=A_{1} u_{n}+A u_{n}^{3}
$$

and by the change of variables $v=\sqrt{|A|} u$ we have

$$
v_{n+1}=A_{1} v_{n} \pm v_{n}^{3} \text {. }
$$

The second iterate map is given by

$$
v_{n+2}=A_{1}\left(A_{1} v_{n} \pm v_{n}^{3}\right) \pm\left(A_{1} v_{n} \pm v_{n}^{3}\right)^{3} .
$$

Removing the terms up to the third order we have

$$
v_{n+2}=A_{1}^{2} v_{n} \pm\left(A_{1}+A_{1}^{3}\right) v_{n}^{3} .
$$

The Taylor expansion of $A_{1}(\boldsymbol{\mu})$ around the point 0 is

$$
A_{1}(\boldsymbol{\mu})=A_{1}(0)+\sum_{i=0}^{p-1} \frac{\partial A_{1}}{\partial \mu_{i}}(0) \mu_{i}
$$

Because $A_{1}(\boldsymbol{\mu})=\frac{\partial \Psi}{\partial z}(0, \boldsymbol{\mu})$ it follows that $\frac{\partial A_{1}}{\partial \mu_{i}}(\boldsymbol{\mu})=$ $\frac{\partial^{2} \Psi}{\partial \mu_{i} \partial z}(0, \boldsymbol{\mu}), i=0,1, \ldots, p-1$. Hence

$$
A_{1}(\boldsymbol{\mu})=-1+\sum_{i=0}^{p-1} \frac{\partial^{2} \Psi}{\partial \mu_{i} \partial z}(0) \mu_{i} .
$$

Let $A_{1}(\boldsymbol{\mu})=-1 \pm \beta$ where

$$
\begin{cases}\beta=\sum_{i=0}^{p-1} \frac{\partial^{2} \Psi}{\partial \mu_{i} \partial z}(0) \mu_{i} & \text { if } \prod_{i=0}^{p-1} \frac{\partial^{2} \Psi}{\partial \mu_{i} \partial z}(0)>0 \\ \beta=-\sum_{i=0}^{p-1} \frac{\partial^{2} \Psi}{\partial \mu_{i} \partial z}(0) \mu_{i} & \text { if } \prod_{i=0}^{p-1} \frac{\partial^{2} \Psi}{\partial \mu_{i} \partial z}(0)<0 .\end{cases}
$$

Notice that under this assumption one has

$$
\frac{\partial^{2} \Psi}{\partial \mu_{0} \partial z}(0) \neq 0, \frac{\partial^{2} \Psi}{\partial \mu_{1} \partial z}(0) \neq 0, \ldots, \frac{\partial^{2} \Psi}{\partial \mu_{p-1} \partial z}(0) \neq 0 .
$$

The single map is now

$$
v_{n+1}=(-1 \pm \beta) v_{n} \pm v_{n}^{3} .
$$

The fixed point $v=0$ of the map representing $v_{n+1}=(-1+\beta) v_{n} \pm v_{n}^{3}$ is stable if $\beta>0$ and unstable if $\beta<0$. Analogously, the fixed point $v=0$ of the map $v_{n+1}=(-1-\beta) v_{n} \pm v_{n}^{3}$ is stable if $\beta<0$ and unstable if $\beta>0$.

The second iterate map is given by

$$
\begin{aligned}
v_{n+2}= & (-1 \pm \beta)^{2} v_{n} \\
& \pm\left((-1 \pm \beta)+(-1 \pm \beta)^{3}\right) v_{n}^{3} .
\end{aligned}
$$

Recall that, our main objective here is to study the dynamics of Eq. (30) near the origin. Hence, we can rewrite the map (30) as

$$
\begin{aligned}
v_{n+1}= & (-1 \pm \beta)^{2} v_{n} \\
& \pm\left((-1 \pm \beta)+(-1 \pm \beta)^{3}\right) v_{n}^{3} .
\end{aligned}
$$

This transformation shifts, in one step, the sequence of point $v_{n}$ near the fixed point, but does not change their values, and thus its dynamics is preserved.

Consider the change of variables $h=$ $\sqrt{\left|(-1 \pm \beta)+(-1 \pm \beta)^{3}\right|}$. Substituting in (31) and simplifying we have

$$
h_{n+1}=h_{n} \pm \gamma h_{n} \pm h_{n}^{3},
$$

where $\gamma=2 \beta+\beta^{2}$ and $-\gamma=-2 \beta+\beta^{2}$. This is precisely the normal form of the pitchfork bifurcation. In fact, if $G(v, \beta)$ is a map representing (31) then 
at the fixed point $(v, \beta)=(0,0)$ one has

$$
\begin{aligned}
\frac{\partial G}{\partial v}(0,0)= & \left((-1 \pm \beta)^{2}\right) \pm 3((-1 \pm \beta) \\
& \left.+(-1 \pm \beta)^{3}\right)\left.v^{2}\right|_{(v, \beta)=(0,0)}=1 \\
\frac{\partial G}{\partial \beta}(0,0)= & \pm 2(-1 \pm \beta) v \\
& \pm\left.\left( \pm 1 \pm 3(-1 \pm \beta)^{2}\right) v^{3}\right|_{(v, \beta)=(0,0)} \\
= & 0 \\
\frac{\partial^{2} G}{\partial \beta \partial v}(0,0)= & \pm 2(-1) \pm 3( \pm 1 \pm 3(-1)) \neq 0
\end{aligned}
$$

and

$$
\frac{\partial^{2} G}{\partial v^{2}}(0,0)=0, \quad \frac{\partial^{3} G}{\partial v^{3}}(0,0)=\mp 12 \neq 0 .
$$

Theorem 4. Let us assume that the map $z_{n+1}=$ $\Psi\left(z_{n}, \mu_{0}, \mu_{1}, \ldots, \mu_{p-1}\right)$ has a nonhyperbolic fixed point at $0 \in \mathbb{R}^{p+1}$ such that $\Psi(0, \boldsymbol{\mu})=0$ for all $\left(\mu_{0}, \mu_{1}, \ldots, \mu_{p-1}\right)$ close to $(0,0, \ldots, 0)$ and $\frac{\partial \Psi}{\partial z}(0)=$ -1 . If

$$
\frac{\partial^{2} \Psi}{\partial \mu_{0} \partial z}(0) \neq 0, \frac{\partial^{2} \Psi}{\partial \mu_{1} \partial z}(0) \neq 0, \ldots, \frac{\partial^{2} \Psi}{\partial \mu_{p-1} \partial z}(0) \neq 0
$$

and

$$
\frac{1}{6} \frac{\partial^{3} \Psi}{\partial z^{3}}(0)+\frac{1}{4}\left(\frac{\partial^{2} \Psi}{\partial z^{2}}(\mathbf{0})\right)^{2} \neq 0
$$

then the normal forms of the map in a small neighborhood of the fixed point 0 are given by

$$
h_{n+1}=h_{n} \pm \gamma h_{n} \pm h_{n}^{3},
$$

and the period-doubling bifurcation takes place at 0.

Since $\frac{\partial \Psi}{\partial y}(0)=-1$, the condition $\frac{1}{6} \frac{\partial^{3} \Psi}{\partial z^{3}}(0)+$ $\frac{1}{4}\left(\frac{\partial^{2} \Psi}{\partial z^{2}}(\mathbf{0})\right)^{2} \neq 0$ is equivalent to the Schwarzian derivative $S \Psi(0) \neq 0$ [Elaydi, 2008].

Notice that Condition (32) must be computed only after shifting the fixed point to the origin. So, in general, we cannot apply directly this test for the initial composition operator $\Phi_{s}$. In order to apply it without any restrictions, we need first to transform our map to the form (21). This is needed because this transformation introduces a new variable $z$ which is a function of $\mu_{0}, \mu_{1}, \ldots, \mu_{p-1}$. Therefore the derivatives $\sum_{i=0}^{p-1} \frac{\partial \Psi}{\partial \mu_{i}} \mu_{i}$ may be changed. For instance consider the map $g\left(y, \mu_{0}, \mu_{1}\right)=y-\left(\mu_{0}+\right.$ $\left.\mu_{1}\right)+\left(\mu_{0}+\mu_{1}\right)^{2}$. So $\sum_{i=0}^{1} \frac{\partial g}{\partial \mu_{i}} \mu_{i}=-\left(\mu_{0}+\mu_{1}\right)+$ $2\left(\mu_{0}+\mu_{1}\right)^{2}$. By the change of variables $z=y-$ $\left(\mu_{0}+\mu_{1}\right)$ it follows that $G\left(z, \mu_{0}, \mu_{1}\right)=z+\left(\mu_{0}+\mu_{1}\right)^{2}$ and therefore $\sum_{i=0}^{1} \frac{\partial G}{\partial \mu_{i}} \mu_{i}=2\left(\mu_{0}+\mu_{1}\right)^{2}$.

Example 7.1. Let us consider the map given in Example 6.2 with $\mu_{n} \in \mathbb{R} \backslash\{0\}$ for all $n=0,1,2, \ldots$ such that

$$
\prod_{i=0}^{p-1} \mu_{i}<0 \text {. }
$$

Hence, the fixed point $x^{*}=0$ is locally asymptotically stable when $\left|\prod_{i=0}^{p-1} \mu_{i}\right|<1$. Let us assume that $\prod_{i=0}^{p-1} \mu_{i}=-1$. It follows that $\Phi_{p}^{\prime}(0)=-1$ and $\frac{\partial \Phi_{p}}{\partial \mu_{i}}(0)=0$, for all $i=0,1, \ldots, p-1$. Moreover, $\frac{\partial^{2} \Phi_{p}}{\partial \mu_{i} \partial x}=-\frac{1}{\mu_{i}} \neq 0$ and

$$
\frac{1}{6} \frac{\partial^{3} \Phi_{p}}{\partial x^{3}}(0)+\frac{1}{4}\left(\frac{\partial^{2} \Phi_{p}}{\partial x^{2}}(0)\right)^{2}=\sum_{i=0}^{p-1} \prod_{j=0}^{i-1} \mu_{j} \neq 0 .
$$

Thus, the fixed point $x^{*}=0$ of $x_{n+1}=H_{n}\left(x_{n}\right)$, $H_{n}=\mu_{n} \sin (x)$, undergoes a period-doubling bifurcation when $\prod_{i=0}^{p-1} \mu_{i}=-1$.

Example 7.2. Consider the one-dimensional Ricker map given in (5). The period-doubling bifurcation equation is given by $\left(1-\bar{x}_{0}\right)\left(1-\bar{x}_{1}\right)=-1$, or equivalently

$$
\left(1-\lambda_{0}-\lambda_{1}+\bar{x}_{0}\right)\left(1-\bar{x}_{0}\right)=-1 .
$$

Equation (33) has the solutions

$$
\begin{aligned}
\bar{x}_{0_{ \pm}} & =\bar{x}_{ \pm}^{p d} \\
& =\frac{1}{2}\left(\lambda_{0}+\lambda_{1} \pm \sqrt{\left(\lambda_{0}+\lambda_{1}\right)\left(\lambda_{0}+\lambda_{1}-4\right)+8}\right) .
\end{aligned}
$$

After shifting the fixed point $\left(\bar{x}_{0}, \lambda_{0}, \lambda_{1}\right)$ to the origin one obtains the equation

$$
\begin{aligned}
y_{n+1}= & \left(y_{n}+\bar{x}_{0}\right) \\
& \times e^{\mu_{0}+\lambda_{0}+\mu_{1}+\lambda_{1}-\left(y_{n}+\bar{x}_{0}\right)\left(1+e^{\mu_{0}+\lambda_{0}-\left(y_{n}+\bar{x}_{0}\right)}\right)} \\
& -\bar{x}_{0} .
\end{aligned}
$$

By the change of variable $z_{n}=y_{n}-Y$, where $Y=\frac{1}{2}\left(-\mu_{1}+\left(1-\bar{x}_{1}\right) \bar{x}_{0} \mu_{0}\right)$, we obtain the equation $z_{n+1}=\Psi\left(z_{n}\right)$, where

$$
\begin{aligned}
\Psi(z)= & \left(z+Y+\bar{x}_{0}\right) \\
& \times e^{\mu_{0}+\lambda_{0}+\mu_{1}+\lambda_{1}-\left(z+Y+\bar{x}_{0}\right)\left(1+e^{\left.\mu_{0}+\lambda_{0}-\left(z+Y+\bar{x}_{0}\right)\right)}\right.} \\
& -Y-\bar{x}_{0} .
\end{aligned}
$$




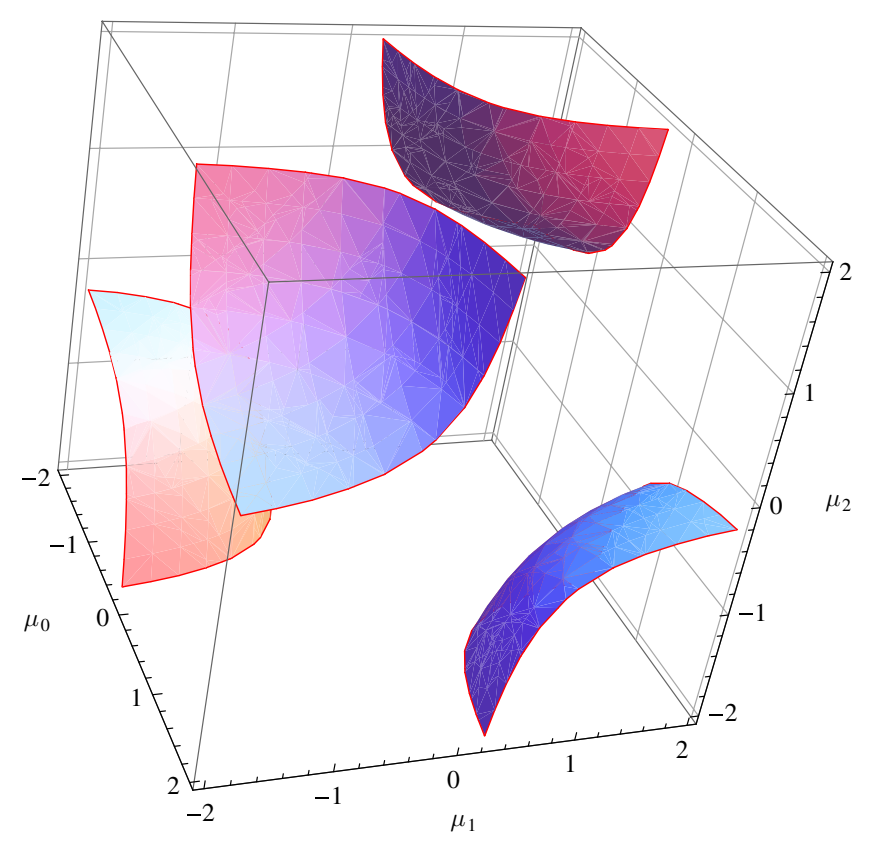

Fig. 3. The bifurcation surface, in the parameter space $\left(\mu_{0}, \mu_{1}, \mu_{2}\right)$, where occurs the period-doubling bifurcation of the map given in Example 7.1.

Hence

$$
\begin{aligned}
\frac{\partial \Psi}{\partial z}(0,0,0)= & \left(1-\bar{x}_{0}\right)\left(1-\bar{x}_{1}\right) \\
= & \left(1-\bar{x}_{0}\right)\left(1-\lambda_{0}-\lambda_{1}+\bar{x}_{0}\right)=-1, \\
\frac{\partial^{2} \Psi}{\partial \mu_{0} \partial z}(0,0,0)= & 4 \bar{x}_{0}^{2}\left(-1+\bar{x}_{1}\right)^{2}-\bar{x}_{0}^{3}\left(-1+\bar{x}_{1}\right)^{2} \\
& +2\left(1-4 \bar{x}_{1}+\bar{x}_{1}^{2}\right) \\
& -2 \bar{x}_{0}\left(2-7 \bar{x}_{1}+3 \bar{x}_{1}^{2}\right),
\end{aligned}
$$

and

$$
\frac{\partial^{2} \Psi}{\partial \mu_{1} \partial z}(0,0,0)=\frac{\begin{array}{c}
2-4 \bar{x}_{0}+\bar{x}_{0}^{2}-\left(4-8 \bar{x}_{0}\right. \\
\left.+3 \bar{x}_{0}^{2}\right) \bar{x}_{1}+\left(\bar{x}_{0}-1\right) \bar{x}_{1}^{2}
\end{array}}{2} .
$$

Since $\bar{x}_{1}=\lambda_{0}+\lambda_{1}-\bar{x}_{0}$, we conclude ${ }^{1}$ that $\frac{\partial^{2} \Psi}{\partial \mu_{0} \partial z}(0,0,0)>0$ when $\bar{x}_{0}=\bar{x}_{0_{+}}^{p d}$ and $\frac{\partial^{2} \Psi}{\partial \mu_{0} \partial z}(0$, $0,0)<0$ when $\bar{x}_{0}=\bar{x}_{0_{-}}^{p d}$, and $\frac{\partial^{2} \Psi}{\partial \mu_{1} \partial z}(0,0,0)<0$ in both situations $\bar{x}_{0}=\bar{x}_{0_{-}}^{p d}$ and $\bar{x}_{0}=\bar{x}_{0_{+}}^{p d}$. Hence

$$
\frac{\partial^{2} \Psi}{\partial \mu_{0} \partial z}(0,0,0) \neq 0 \quad \text { and } \quad \frac{\partial^{2} \Psi}{\partial \mu_{1} \partial z}(0,0,0) \neq 0 .
$$

Similar techniques allow us to show that $\frac{1}{6} \frac{\partial^{3} \Psi}{\partial z^{3}}(0,0,0)+\frac{1}{4}\left(\frac{\partial^{2} \Psi}{\partial z^{2}}(0,0,0)\right)^{2}>0$ in both situations $\bar{x}_{0}=\bar{x}_{0_{-}}^{p d}$ and $\bar{x}_{0}=\bar{x}_{0_{+}}^{p d}$.

Substituting the values of the bifurcation solutions in (6) yields two equations of the form

$$
\lambda_{0}+\lambda_{1}-y=y e^{\lambda_{0}-y}
$$

where $y$ is given by $\bar{x}_{+}^{p d}$ or $\bar{x}_{-}^{p d}$.

Once again we invoke the implicit function theorem [Protter \& Morrey, 1991; Zeidler, 1993] in order to guarantee the existence of solutions of Eq. (34). In Fig. 1 implicitly, in the parameter space $\lambda_{0}$ and $\lambda_{1}$, these exact solutions (black curves) are represented. If the parameters $\lambda_{0}$ and $\lambda_{1}$ are on the black curves, then the period-doubling bifurcation takes place. Note that the period-doubling bifurcation curves intersect the axes at the points $(2.53039,0)$ and $(0,2.53039)$ and they intersect themselves at the point $(2.52647,2.52647)$. Moreover, if $\lambda_{0}=\lambda_{1}$, i.e. if the parameters are on the diagonal line, the equation loses its periodicity (in other words, the map is autonomous).

Using computer assistance we present in Fig. 4 the bifurcation scenario of the two-periodic cycle in the parameter space $\left(\lambda_{0}, \lambda_{1}\right)$. In region $A_{1}$, the

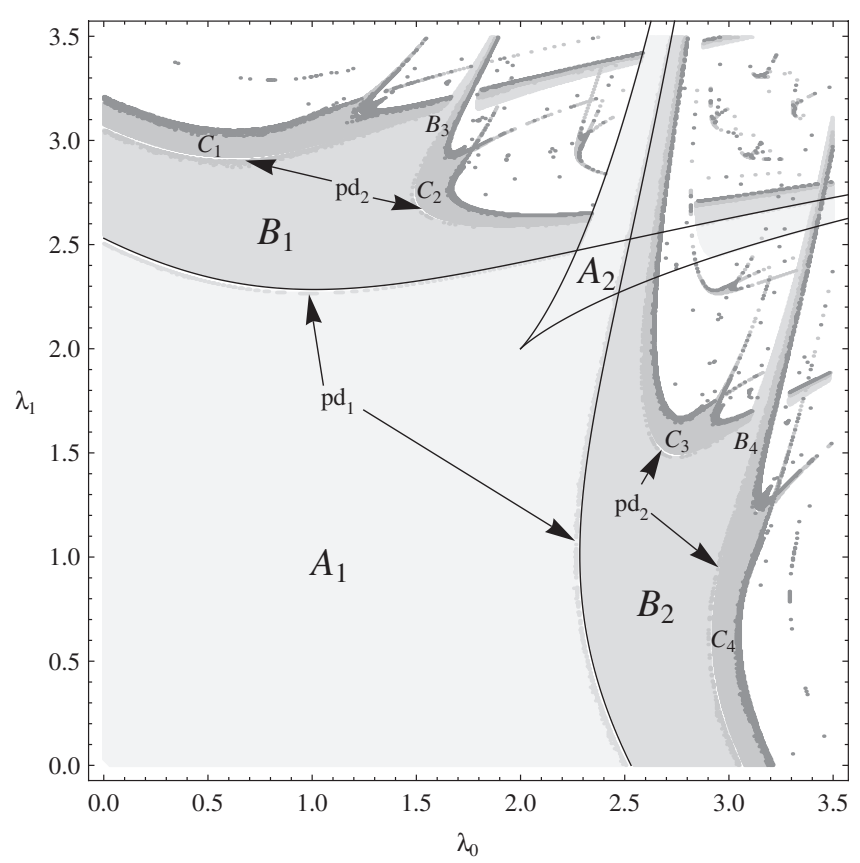

Fig. 4. The bifurcation scenario in the parameter space of the two-periodic Ricker map.

\footnotetext{
${ }^{1}$ We omit the computations here since they are long.
} 
two-periodic Ricker map has an asymptotically stable two-periodic cycle. ${ }^{2}$ The period-doubling bifurcation curves are represented by $p d_{1}$. Hence, as $\lambda_{0}$ and $\lambda_{1}$ pass these two curves, a perioddoubling bifurcation is seen. The two-periodic cycle become unstable and a new asymptotically stable four-periodic cycle is born. This new fourperiodic cycle is always asymptotically stable whenever the parameters are in the region $B_{1} \cup B_{2}$. This four-periodic cycle loses its stability whenever the parameters pass the curves $p d_{2}$. These curves undergo a period-doubling bifurcation. Consequently, the four-periodic cycle becomes unstable and a new asymptotically stable eight-periodic cycle is created. The stability region of the eight-periodic cycle is given by $\bigcup_{i=1}^{4} C_{i}$. Again, this cycle loses its stability whenever the parameters pass the curves $p d_{3}$ in which a period-doubling bifurcation occurs. This period-doubling scenario continues route to chaos.

If the parameters are in region $A_{2}$, then the two-periodic Ricker map has three two-periodic cycles. Two of them are asymptotically stable and the third is unstable. In region $B_{3} \cup B_{4}$, the twoperiodic Ricker map has two asymptotically stable four-periodic cycles and one unstable four-periodic cycle.

\section{References}

AlSharawi, Z. \& Angelos, J. [2006] "On the periodic logistic equation," Appl. Math. Comput. 180, 342352.

Arrowsmith, D. K. \& Place, C. M. [1990] An Introduction to Dynamical Systems (Cambridge University Press, Cambridge).

Crawford, J. D. [1991] "Introduction to bifurcation theory," Rev. Mod. Phys. 63, 991-1037.

Cushing, J. [1998] "Periodically forced nonlinear systems of difference equations," J. Diff. Eqs. Appl. 3, 547561.

Cushing, J., Costantino, R., Dennis, B., Desharnais, R. \& Henson, S. [2003] Chaos in Ecology: Experimental Nonlinear Dynamics, 1st edition, Theoretical Ecology Series (Academic Press).

D'Aniello, E. \& Oliveira, H. [2009] "Pitchfork bifurcation for nonautonomous interval maps," J. Diff. Eqs. Appl. 15, 291-302.

Dhooge, A., Govaerts, W., Kuznetsov, Yu. A., Meijer, E. \& Sautois, B. [2008] "New features of the software MatCont for bifurcation analysis of dynamical systems," Math. Comput. Model. Dyn. Syst. 14, 147-175.

Elaydi, S. \& Sacker, R. [2005a] "Global stability of periodic orbits of nonautonomous difference equations and populations biology," J. Diff. Eqs. 208, 258273.

Elaydi, S. \& Sacker, R. [2005b] "Nonautonomous Beverton-Holt equations and the Cushing-Henson conjectures," J. Diff. Eqs. Appl. 11, 337-346.

Elaydi, S. \& Sacker, R. [2005c] "Skew-product dynamical systems: Applications to difference equations," Proc. Second Ann. Celebration of Mathematics (United Arab Emirates).

Elaydi, S. \& Sacker, R. [2006] "Periodic difference equations, population biology and the Cushing-Henson conjectures," Math. Biosci. 201, 195-207.

Elaydi, S. [2008] Discrete Chaos: With Applications in Science and Engineering, 2nd edition (Chapman and Hall/CRC).

Elaydi, S., Luís, R. \& Oliveira, H. [2011] "Towards a theory of periodic difference equations and its application to population dynamics," Dynamics, Games and Science I, DYNA 2008, Springer, Series: Springer Proceedings in Mathematics, Vol. 1, pp. 287-321.

Grinfeld, M., Knight, P. A. \& Lamba, H. [1996] "On the periodically perturbed logistic equation," J. Phys. A: Math. Gen. 29, 8035-8040.

Hale, J. K. \& Koçak, H. [1991] Dynamics and Bifurcations, Texts in Applied Mathematics, Vol. 3 (Springer, Berlin).

Hamaya, Y. [2004] "Bifurcation of almost periodic solutions in difference equations," J. Diff. Eqs. Appl. 10, 257-297.

Henson, S. [1996] "Existence and stability of nontrivial periodic solutions of periodically forced discrete dynamical systems," J. Diff. Eqs. Appl. 2, 315-331.

Hüls, T. [2007] "A model function for non-autonomous bifurcations of maps," Discr. Contin. Dyn. Syst. (Series B) 2, 351-363.

Iooss, G. [1979] "Bifurcations of maps and applications," Mathematics Studies (North-Holland, Amsterdam, NY, Oxford), Université de Nice, France.

Kloeden, P., Pötzsche, C. \& Rasmussen, M. [2011a] "Discrete-time nonautonomous dynamical systems," manuscript.

Kloeden, P. \& Rasmussen, M. [2011b] Nonautonomous Dynamical Systems, Mathematical Surveys and Monographs, Vol. 176 (AMS, Providence, RI).

Kuznetsov, Y. [2004] Elements of Applied Bifurcation Theory, Applied Mathematics Sciences, Vol. 112 (Springer, Berlin).

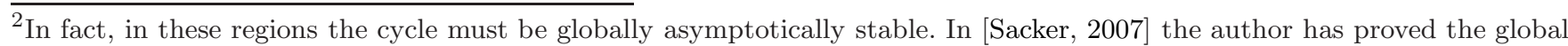
stability of the cycle for the $p$-periodic Ricker equation when $\lambda_{i} \in(0,2), i=0,1, \ldots, p-1$.
} 
Luís, R., Elaydi, S. \& Oliveira, H. [2010] "Nonautonomous periodic systems with Allee effects," J. Diff. Eqs. Appl. 16, 1179-1196.

Panfilov, A. [2005] "Non-linear dynamical systems: Theoretical biology and bioinformatics," Technical report (Utrecht University, Utrecht).

Pötzsche, C. [2010a] "Geometric theory of discrete nonautonomous dynamical systems," Lecture Notes in Mathematics, Vol. 2002 (Springer, Berlin).

Pötzsche, C. [2010b] "Nonautonomous bifurcation of bounded solutions I: A Lyapunov-Schmidt approach," Discr. Contin. Dyn. Syst. (Series B) 14, 739-776.

Pötzsche, C. [2011a] "Nonautonomous bifurcation of bounded solutions II: A shovel bifurcation pattern," Discr. Contin. Dyn. Syst. (Series A) 3, 941-973.

Pötzsche, C. [2011b] "Bifurcations in nonautonomous dynamical systems: Results and tools in discrete time," Proc. Int. Workshop: Future Directions in Difference Equations, Vigo, Spain, pp. 163-212.

Pötzsche, C. [2012] "Bifurcations in a periodic discretetime environment," Nonlin. Anal.: Real World Appl. 14, 53-82.

Protter, M. H. \& Morrey, C. B. [1991] A First Course in Real Analysis, 2nd edition (Springer-Verlag, NY).
Rasmussen, M. [2006] "Towards a bifurcation theory for nonautonomous difference equation," J. Diff. Eqs. Appl. 12, 297-312.

Rasmussen, M. [2007] Attractivity and Bifurcation for Nonautonomous Dynamical Systems, Lecture Notes in Mathematics, Vol. 1907 (Springer, Berlin).

Robinson, C. [1999] Dynamical Systems: Stability, Symbolic Dynamics, and Chaos, 2nd edition (Inc., Boca Raton, CRC Press).

Sacker, R. [2007] "A note on the periodic Ricker map," J. Diff. Eqs. Appl. 13, 89-92.

Sacker, R. \& Sell, J. [1977] Lifting Properties in SkewProduct Flows with Applications to Differential Equations, AMS Memoirs, Vol. 11, 67 pp.

Wiggins, S. [2003] Introduction to Applied Nonlinear Dynamical Systems and Chaos (Springer-Verlag, NY, Berlin-Heidelberg).

Yet, N. T., Doan, T. S., Jäger, T. \& Siegmund, S. [2011] "Nonautonomous saddle-node bifurcations in the quasiperiodically forced logistic map," Int. J. Bifurcation and Chaos 21, 1427-1438.

Zeidler, E. [1993] Nonlinear Functional Analysis and Its Applications I (Fixed-Points Theorems) (Springer, Berlin). 